\title{
Theory of proximity effect in two-dimensional unconventional superconductor with Rashba spin-orbit interaction
}

\author{
Shun Tamura ${ }^{1}$, Yukio Tanaka ${ }^{1}$ \\ 1 Department of Applied Physics, \\ Nagoya University, Nagoya 464-8603, Japan
}

\begin{abstract}
We study the anomalous proximity effect in diffusive normal metal (DN)/unconventional superconductor junctions, where the local density of states (LDOS) in the DN has a zero-energy peak due to the penetration of the odd-frequency spin-triplet $s$-wave pairing. In this study, we consider a two-dimensional unconventional superconductor on the substrate in the presence of a Rashba spinorbit coupling (RSOC) $\lambda$, where the Rashba vector is parallel to the $z$-direction. The anomalous proximity effect, originally predicted in spin-triplet $p$-wave superconductor junctions, is sensitive to the RSOC. It disappears with the increase of $\lambda$. On the other hand, the anomalous proximity effect can be switched on by the large $\lambda$ values in the spin-singlet $d_{x y}$-wave superconductor junctions. The resulting zero-energy LDOS and the magnitude of the odd-frequency spin-triplet $s$-wave pair amplitude increase with the increase of $\lambda$.
\end{abstract}

\section{INTRODUCTION}

In unconventional superconductors (SCs), nodes of the energy gap and the change of the sign of the pair potential (gap function) are generated on the Fermi surface. It is known that the change of the sign of the pair potential produces in-gap zero-energy states localized near the surface, known as zero-energy surface Andreev bound states (ZESABSs) [1 5]. The ZESABS manifests itself as a zero bias conductance peak in quasiparticle tunneling experiments [4, 6 9].

Nowadays, it is recognized that the ZESABS has deep physical meanings from the aspects of topology and symmetry in condensed matter physics [10]. It is known that the ZESABS is protected by the topological invariant defined in the bulk Hamiltonian[10-15]. Thus, unconventional superconductors hosting nodes can be classified as topological superconductors 13, 16, 17]. Besides this property, the ZESABS has a significant meaning from the aspect of the symmetry of Cooper pairs.

The breaking of translational invariance induces an odd-frequency pairing, i.e., spin-singlet odd-parity or spin-triplet even-parity, even if the symmetry of the bulk superconductor has a standard even-frequency pairing, i.e., spin-singlet even-parity or spin-triplet oddparity [10, 18, 19], due to the mixing of parity 20 24]. Thus, the ZESABS always accompanies an oddfrequency pairing due to the breaking of translational invariance in the superconductor $20-22]$. If the symmetry of the bulk superconductor is a spin-singlet $d_{x y}$-wave, the resulting ZESABSs have an odd-frequency spin-singlet odd-parity (OSO, see Table@) pairing component [20, 22]. On the other hand, if the symmetry of the bulk superconductor is a spin-triplet $p$-wave, the resulting ZESABSs exhibit an odd-frequency spin-triplet even-parity (OTE, see Table I) pairing symmetry [20, 22, 25]. In the latter case, if the superconductor is contacted by a diffusive normal metal (DN), the OTE pairing can penetrate into the DN [25], as it has an $s$-wave component, which is ro-
TABLE I. Possible pairing symmetry and symbols for symmetry of Cooper pair amplitude. Pairing symmetries of Cooper pair are classified into ESE (even-frequency spinsinglet even-parity), ETO (even-frequency spin-triplet oddparity), OSO (odd-frequency spin-singlet odd-parity), and OTE (odd-frequency spin-triplet even-parity).

\begin{tabular}{|c|c|c|c|}
\hline frequency & spin & parit & symbol \\
\hline Even & Singlet & Even & ESE \\
\hline Even & Triplet & Odd & ETO \\
\hline Odd & Singlet & Odd & OSO \\
\hline Odd & Triplet & Even & OTE \\
\hline
\end{tabular}

bust against impurity scattering. The resulting local density of states (LDOS) in the DN has a zero-energy peak (ZEP) 25-29] in contrast to the conventional proximity effect, where the LDOS has a gap-like structure around zero-energy [30]. This unusual condition is known as an anomalous proximity effect $26-28,31$. The anomalous proximity effect can occur in a $\mathrm{DN} /$ noncentrosymmetric superconductor junction when the spin-triplet pair potential is dominant [32].

The anomalous proximity effect, triggered by an oddfrequency spin-triplet $s$-wave pairing, shows several interesting physical properties: i) ZEP of the LDOS in the DN 26, 27, 33, 34], ii) ZEP of the LDOS at rough surface [35], iii) zero bias conductance peak in quasiparticle transport in the $\mathrm{DN}$ /spin-triplet $p$-wave superconductor junctions [26, 27, 31], iv) significant enhancement of the Josephson current at low temperatures in the DN/spintriplet $p$-wave superconductor junctions [28] v) paramagnetic Meissner response [36-43], and vi) anomalous surface impedance [4, 45]. It has been also shown that the anomalous proximity effect can occur in topologically designed hybrid systems based on conventional spin-singlet $s$-wave superconductor systems with spin-orbit coupling and Zeeman effect [31, 46, 47] and the anomalous proximity effect has been studied considering the classifica- 
tion of the topological nature of the Hamiltonian [48]. In addition, there are several studies reporting that oddfrequency pairings appear as a Majorana fermion, which is a special type of the ZESABS in various topological superconducting systems [46, 49 59].

Although OTE $s$-wave pairing has been discussed in diffusive ferromagnet (DF)/conventional spin-singlet $s$ wave superconductor junctions, it is difficult to realize pure OTE $s$-wave pairing state in a DF $60-71]$. Conversely, the demonstrated anomalous proximity effect generated by ZESABSs specific to unconventional superconductors is remarkable, as it can induce a purely odd-frequency pairing or at least a significant amount of it at low energy. Thus, it is interesting to understand the mechanism of effect of several external perturbations on the anomalous proximity effect. Among these, the Rashba spin-orbit coupling is very interesting, as it inevitably exists near the interface or the thin films of superconductor grown on the substrate. Here, we focus on the effect of the Rashba spin-orbit coupling (RSOC) on the anomalous proximity effect. As shown by a study of noncentrosymmetric superconductors, the RSOC can mix spin-triplet odd-parity pairing with the spin-singlet even-parity pairing $[72,80]$. It is possible that the anomalous proximity effect can be induced even in a spin-singlet $d_{x y}$-wave superconductor, when an OTE $s$-wave pairing can be induced from the RSOC.

In this paper, we study the anomalous proximity effect of DN/superconductor junctions with the RSOC by choosing a spin-triplet $p_{x}$-wave pairing with a $\mathbf{d}$-vector parallel to $z$-axis and a spin-singlet $d_{x y}$-wave pairing. As the RSOC, we consider that it is proportional to $\mathbf{g} \cdot \boldsymbol{\sigma}$ with $\mathbf{g}=\left(\sin k_{y},-\sin k_{x}, 0\right)$ which is originated from the inversion symmetry breaking in the $z$-direction and Pauli matrix $\boldsymbol{\sigma}$ in the spin-space. We calculate the surface density of states, the spin-triplet $s$-wave component of the odd-frequency pairing, and the local density of states in the DN attached to superconductors, based on a tight-binding model. For the spin-triplet $p_{x}$-wave superconductor, as the ZESABS is fragile against the RSOC [17, 81], the magnitude of the OTE pairing is reduced and the resulting anomalous proximity effect is weakened by the RSOC. On the other hand, it is shown that the anomalous proximity effect can be switched on by the RSOC for spin-singlet $d_{x y}$-wave superconductor junctions. The magnitude of the LDOS at zero-energy in the DN increases with the increase of the RSOC. This indicates that the anomalous proximity effect can be detected for high $T_{\mathrm{c}}$ cuprate junctions.

The organization of this paper is as follows. In Section III, we discuss the momentum resolved surface density of states and the OTE pairing for semi-infinite superconductors with the RSOC. In Section [II] we consider the DN/superconductor junctions and discuss the LDOS and the OTE pairing in the DN. In Section IV] we summarize our results.

\section{SEMI-INFINITE SUPERCONDUCTOR}

(a) Bulk without RSOC

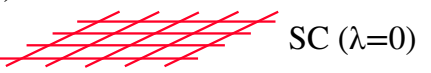

(b) Bulk with RSOC

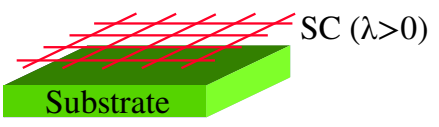

(c) Semi-infinite system or junction without RSOC

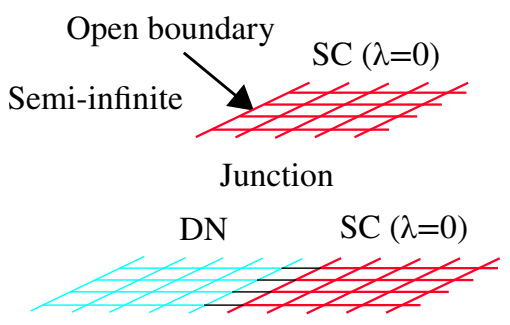

FIG. 1. Schematic pictures corresponding to Table II (a) Bulk system without RSOC, (b) Bulk system with RSOC, (c) Semi-infinite system or junction without RSOC.

TABLE II. Possible symmetry of Cooper pair amplitude for spin-triplet $p_{x}$-wave superconductor and spin-singlet $d_{x y}$-wave superconductor in the bulk system. TS indicates translational symmetry in the $x$-direction. Symbols in the third and fourth columns are given in Table I (a), (b) and (c) in the first column correspond to those in Fig. 1.

\begin{tabular}{|c|c|c|c|c|}
\hline & $\mathrm{TS}$ & RSOC & $\begin{array}{c}\text { spin-triplet } \\
p_{x} \text {-wave }\end{array}$ & $\begin{array}{c}\text { spin-singlet } \\
d_{x y} \text {-wave }\end{array}$ \\
\hline (a) & YES & $\mathrm{NO}$ & ETO & ESE \\
\hline (b) & YES & YES & ETO, ESE & ESE, ETO \\
\hline (c) & NO & $\mathrm{NO}$ & ETO, OTE & ESE, OSO \\
\hline
\end{tabular}

Before we start discussion, we explain about possible symmetry class of Cooper pair by the external symmetry breaking for spin-triplet $p_{x}$-wave and spin-singlet $d_{x y^{-}}$ wave superconductors. Pairing symmetry of the spintriplet $p_{x}$-wave superconductor in the bulk belongs to even-frequency spin-triplet odd-parity (ETO) as shown in Fig. 1 (a) and Table [I (a). In the presence of the RSOC, since the spin-rotational symmetry and spatial parity are broken at the same time, even-frequency spinsinglet even-parity (ESE) pairing mixes. Then, the resulting pairing symmetry are ESE and ETO [Fig. 1 (b) and Table I (b)] [75]. When only the translational invariance in the $x$-direction is broken, the symmetry of pair amplitude are ETO and OTE shown in Fig. 1 (c) and Table $\amalg(\mathrm{c})$ [10].

On the other hand, the spin-singlet $d_{x y}$-wave superconductor in the bulk belongs to the even-frequency spinsinglet even-parity (ESE) pairing as shown in Fig. 1 (a) 
and Table (a). In the presence of the RSOC, ETO pairing mixes and the resulting pairing symmetry are ESE and ETO [Fig. 1 (b) and Table I (b)] 75]. On the other hand, when only the translational invariance is broken without the RSOC, the symmetry of pair amplitude are ESE and OSO [Fig. 1 (c) and Table II (c)] [10].

In the case that the system does not have translational symmetry in the presence of the RSOC, all pairing symmetries: ETO, ESE, OTE and OSO exist both for spintriplet $p_{x}$-wave and spin-singlet $d_{x y}$-wave superconductors.

\section{A. Model}

We consider two dimensional superconductors on a square lattice model with open boundary condition [Fig. 2] where the superconductor is located in $j_{x} \geq 1$ $\left[\mathbf{j}=\left(j_{x}, j_{y}\right)\right.$ is the coordinate of a lattice site. $]$ and discuss the RSOC dependence of a surface density of states and the odd-frequency spin-triplet $s$-wave pair amplitude. We compare the results for the spin-triplet $p_{x^{-}}$ wave superconductor and the spin-singlet $d_{x y}$-wave one. Throughout this paper, we use lattice constant as a unit of length. The Hamiltonian $\mathcal{H}_{\mathrm{SC}}$ is given by

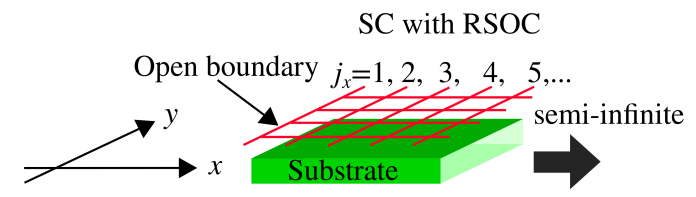

FIG. 2. Schematic illustration of semi-infinite lattice model $\left(j_{x} \geq 1\right)$ SC with RSOC.

$$
\begin{aligned}
& \mathcal{H}_{\mathrm{SC}}=\mathcal{H}_{t}+\mathcal{H}_{\mathrm{SO}}+\mathcal{H}_{\Delta}-\mu \sum_{\mathbf{j}} n_{\mathbf{j}}, \\
& \mathcal{H}_{t}=-t \sum_{\langle\mathbf{i}, \mathbf{j}\rangle, \sigma}\left(c_{\mathbf{i}, \sigma}^{\dagger} c_{\mathbf{j}, \sigma}+\text { H.c. }\right) \\
& \mathcal{H}_{\mathrm{SO}}=\frac{\lambda}{2 i} \sum_{\mathbf{j}, \sigma, \sigma^{\prime}}\left(c_{\mathbf{j}, \sigma}^{\dagger} c_{\mathbf{j}+\mathbf{e}_{y}, \sigma^{\prime}}-c_{\mathbf{j}, \sigma}^{\dagger} c_{\mathbf{j}-\mathbf{e}_{y}, \sigma^{\prime}}\right)\left(\sigma_{1}\right)_{\sigma, \sigma^{\prime}} \\
& -\frac{\lambda}{2 i} \sum_{\mathbf{j}, \sigma, \sigma^{\prime}}\left(c_{\mathbf{j}, \sigma}^{\dagger} c_{\mathbf{j}+\mathbf{e}_{x}, \sigma^{\prime}}-c_{\mathbf{j}, \sigma}^{\dagger} c_{\mathbf{j}-\mathbf{e}_{x}, \sigma^{\prime}}\right)\left(\sigma_{2}\right)_{\sigma, \sigma^{\prime}} \\
& \mathcal{H}_{\Delta}=\sum_{\mathbf{i}, \mathbf{j}, \sigma, \sigma^{\prime}} \Delta_{\mathbf{i}, \mathbf{j}}^{\sigma, \sigma^{\prime}}\left(c_{\mathbf{i}, \sigma}^{\dagger} c_{\mathbf{j}, \sigma^{\prime}}^{\dagger}+\text { H.c. }\right) \\
& n_{\mathbf{j}}=\sum_{\sigma} c_{\mathbf{j}, \sigma}^{\dagger} c_{\mathbf{j}, \sigma}
\end{aligned}
$$

where $\langle\mathbf{i}, \mathbf{j}\rangle$ in Eq. (2) denotes a sum of nearest-neighbor pairs, $\sigma_{i}(i=1,2,3)$ is a Pauli matrix, and $\mathbf{e}_{x}\left(\mathbf{e}_{y}\right)$ is a unit vector in the $x(y)$-direction i.e., $\mathbf{e}_{x}=(1,0)$ and $\mathbf{e}_{y}=(0,1) . t$ is a hopping integral, $\lambda$ is a Rashba spinorbit coupling and $\mu$ is a chemical potential. Here we assume $0 \leq \lambda$. We set $\mu / t=-2.4$ where we do not have to take into account the effect of the van Hove singularity. As the symmetry of the superconducting gap, we consider a spin-triplet $p_{x}$-wave pairing and a spin-singlet $d_{x y}$-wave one. For the spin-triplet $p_{x}$-wave case, the pair potential is

$$
\Delta_{\mathbf{i}, \mathbf{j}}^{\sigma, \sigma^{\prime}}=\frac{\Delta_{0}}{i} \delta_{\mathbf{i}, \mathbf{j}-\mathbf{e}_{x}} \delta_{\sigma,-\sigma^{\prime}}
$$

where we choose the direction of the $\mathbf{d}$-vector parallel to the $z$-direction. For the spin-singlet $d_{x y}$-wave case, the pair potential is

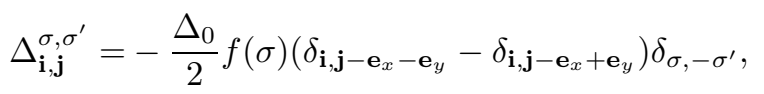

with $f(\uparrow)=1$ and $f(\downarrow)=-1$. We set $\Delta_{0} / t=0.01$ for both spin-triplet $p_{x}$-wave and spin-singlet $d_{x y}$-wave superconductor throughout this paper.

The energy dispersion of the periodic system for the spin-triplet $p_{x}$-wave superconductor is

$$
E(\mathbf{k})= \pm\left|\sqrt{\xi_{\mathbf{k}}^{2}+\left(\Delta_{0}\right)^{2} \sin ^{2} k_{x}} \pm \lambda \sqrt{\sin ^{2} k_{x}+\sin ^{2} k_{y}}\right|
$$

with $\xi_{\mathbf{k}}=-2 t\left(\cos k_{x}+\cos k_{y}\right)-\mu$ and the superconducting gap closes for $\lambda \geq \Delta_{0}$. For the spin-singlet $d_{x y}$-wave superconductor, the energy dispersion is

$$
\begin{aligned}
& E(\mathbf{k}) \\
= & \pm \sqrt{\left[\xi_{\mathbf{k}} \pm \lambda\left(\sin k_{x}+\sin k_{y}\right)\right]^{2}+\left(\Delta_{0} \sin k_{x} \sin k_{y}\right)^{2}}
\end{aligned}
$$

\section{B. Method to calculate local Green's function}

The surface DOS $\rho\left(E, k_{y}\right)$ and the odd-frequency spintriplet $s$-wave pair amplitude are calculated by using a surface Green's function $G_{j_{x}=1}^{\infty}\left(z, k_{y}\right)$. Here, $G_{j_{x}=1}^{\infty}\left(z, k_{y}\right)$ is the Green's function at the leftmost site [Fig. 2]. The derivation of $G_{j_{x}=1}^{\infty}\left(z, k_{y}\right)$ is explained in the Appendix A.

The momentum resolved surface DOS is obtained as

$$
\rho\left(E, k_{y}\right)=-\frac{1}{\pi} \operatorname{Im}\left[\operatorname{tr} G_{j_{x}=1}^{\infty}\left(z=E+i \eta, k_{y}\right)\right]
$$

where $\eta$ is an infinitesimally small constant (we set $\eta / t=$ $10^{-5}$.) and trace is only taken in particle space. Then the surface DOS $\bar{\rho}(E)$ is given by

$$
\bar{\rho}(E)=\frac{1}{2 \pi} \int_{-\pi}^{\pi} d k_{y} \rho\left(E, k_{y}\right) .
$$

The odd-frequency spin-triplet $s$-wave pair amplitude 
at the surface is defined as

$$
\begin{aligned}
P_{S_{z}=1}^{\text {triplet }}\left(\omega_{n}\right) & =P_{\uparrow, \uparrow}\left(\omega_{n}\right), \\
P_{S_{z}=0}^{\text {triplet }}\left(\omega_{n}\right) & =\frac{1}{\sqrt{2}}\left[P_{\uparrow, \downarrow}\left(\omega_{n}\right)+P_{\downarrow, \uparrow}\left(\omega_{n}\right)\right], \\
P_{S_{z}=-1}^{\text {triplet }}\left(\omega_{n}\right) & =P_{\downarrow, \downarrow}\left(\omega_{n}\right), \\
P^{\text {triplet }}\left(\omega_{n}\right) & =\sum_{S_{z}=-1,0,1}\left|P_{S_{z}}^{\text {triplet }}\left(\omega_{n}\right)\right|,
\end{aligned}
$$

with

$$
\begin{aligned}
P_{\sigma, \sigma^{\prime}}\left(\omega_{n}\right) & =\frac{1}{N_{k_{y}}} \sum_{k_{y}} \hat{F}_{\sigma, \sigma^{\prime}}\left(z=i \omega_{n}, k_{y}\right), \\
G_{j_{x}=1}^{\infty}\left(z, k_{y}\right) & =\left(\begin{array}{cc}
\hat{G}_{\sigma, \sigma^{\prime}}\left(z, k_{y}\right) & \hat{F}_{\sigma, \sigma^{\prime}}\left(z, k_{y}\right) \\
\tilde{F}_{\sigma, \sigma^{\prime}}\left(z, k_{y}\right) & \tilde{G}_{\sigma, \sigma^{\prime}}\left(z, k_{y}\right)
\end{array}\right),
\end{aligned}
$$

where $\omega_{n}$ is a Matsubara frequency. In Eq. (17), $\hat{G}_{\sigma, \sigma^{\prime}}\left(z, k_{y}\right), \hat{F}_{\sigma, \sigma^{\prime}}\left(z, k_{y}\right), \tilde{F}_{\sigma, \sigma^{\prime}}\left(z, k_{y}\right)$ and $\tilde{G}_{\sigma, \sigma^{\prime}}\left(z, k_{y}\right)$ are $2 \times 2$ matrices. $\hat{F}_{\sigma, \sigma^{\prime}}\left(z, k_{y}\right)$ and $\tilde{F}_{\sigma, \sigma^{\prime}}\left(z, k_{y}\right)$ are anomalous Green's functions. Note that $P_{S_{z}}^{\text {triplet }}\left(\omega_{n}\right)$ is odd in $\omega_{n}$.

\section{Topological number}

In this subsection, we consider a bulk system and explain about a winding number $W$ and one-dimensional $\mathbf{Z}_{2}$ topological number $\nu$. $W$ and $\nu$ are topological numbers defined in a bulk and they predict the number of the ZESABSs known as bulk-boundary correspondence. The bulk Hamiltonian for the spin-triplet $p_{x}$-wave superconductor and that for the spin-singlet $d_{x y}$-wave superconductor are given by

$$
\begin{aligned}
& H_{p}(\mathbf{k})=H_{t}(\mathbf{k})+H_{\mathrm{SO}}(\mathbf{k})+H_{\Delta}^{p}(\mathbf{k}), \\
& H_{d}(\mathbf{k})=H_{t}(\mathbf{k})+H_{\mathrm{SO}}(\mathbf{k})+H_{\Delta}^{d}(\mathbf{k}),
\end{aligned}
$$

with

$$
\begin{aligned}
H_{t}(\mathbf{k}) & =\left[-2 t\left(\cos k_{x}+\cos k_{y}\right)-\mu\right] \sigma_{0} \tau_{3}, \\
H_{\mathrm{SO}}(\mathbf{k}) & =\lambda\left(\sin k_{y} \sigma_{1} \tau_{0}-\sin k_{x} \sigma_{2} \tau_{3}\right), \\
H_{\Delta}^{p}(\mathbf{k}) & =\Delta_{0} \sin k_{x} \sigma_{1} \tau_{1}, \\
H_{\Delta}^{d}(\mathbf{k}) & =-\Delta_{0} \sin k_{x} \sin k_{y} \sigma_{2} \tau_{2},
\end{aligned}
$$

where $\tau_{i}(i=1,2,3)$ is a Pauli matrix in the particle hole space and $\tau_{0}$ is an identity matrix. Here $H_{t}(\mathbf{k})$ and $H_{\mathrm{SO}}(\mathbf{k})$ are the Fourier transformed form of Eq. (2) and Eq. (3), respectively and $H_{\Delta}^{p}(\mathbf{k})$ and $H_{\Delta}^{d}(\mathbf{k})$ are the Fourier transformed form of Eq. (4) with Eq. (6) and Eq. (7), respectively.

\section{Spin-triplet $p_{x}$-wave superconductor}

In this subsection, we explain about the Topological numbers for the spin-triplet $p_{x}$-wave superconductor.
The winding number can be defined in a bulk if there is a chiral operator $\Gamma$ which anticommutes with the bulk Hamiltonian $(\{H(\mathbf{k}), \Gamma\}=0)$. The winding number is given by

$$
W\left(k_{\|}\right)=\frac{i}{4 \pi} \int_{-\pi}^{\pi} d k_{\perp} \operatorname{Tr}\left[\Gamma H^{-1}(\mathbf{k}) \partial_{k_{\perp}} H(\mathbf{k})\right],
$$

where $k_{\|}=k_{y}$ and $k_{\perp}=k_{x}$ are wave numbers which are parallel and perpendicular to the surface, respectively.

Without the RSOC, the chiral operator is $\Gamma=\Gamma_{p} \equiv$ $S_{z} C T=i \sigma_{1} \tau_{2}\left(S_{z}=\sigma_{3} \tau_{3}\right.$ is a spin-rotational operator around the $z$-axis, $C=\sigma_{0} \tau_{1} K$ is a charge conjugation operator, $T=i \sigma_{2} \tau_{0} K$ is a time-reversal operator and $K$ is a complex conjugation operator.) and it anticommutes with $H_{t}(\mathbf{k})$ and $H_{\Delta}^{p}(\mathbf{k})$ :

$$
\left\{H_{t}(\mathbf{k}), \Gamma_{p}\right\}=\left\{H_{\Delta}^{p}(\mathbf{k}), \Gamma_{p}\right\}=0 .
$$

On the other hand, the RSOC term of the Hamiltonian does not anticommutes with $\Gamma_{p}$ :

$$
\left\{H_{\mathrm{SO}}(\mathbf{k}), \Gamma_{p}\right\} \neq 0 \text {. }
$$

Therefore, the spin-triplet $p_{x}$-wave superconductor with non-zero value of the RSOC does not have chiral symmetry and the winding number cannot be defined. More precisely, the Hamiltonian Eq. (18) anticommutes with $\sigma_{2} \tau_{1}$ but the winding number is always zero regardless of the value of the RSOC and it is not related to the number of the surface states. Then the ZESABSs are fragile against the RSOC given by Eq. (21) [17, 81]. This result does not depend on the direction of the $\mathbf{d}$-vector. The winding number for the spin-triplet $p_{x}$-wave superconductor without the RSOC is

$$
W\left(k_{y}\right)= \begin{cases}0 & k_{y}<-\alpha_{-}^{p}, \\ -2 & -\alpha_{-}^{p}<k_{y}<\alpha_{-}^{p}, \\ 0 & \alpha_{-}^{p}<k_{y},\end{cases}
$$

for $-4 t<\mu<0$ and

$$
W\left(k_{y}\right)= \begin{cases}-2 & -\pi<k_{y}<-\alpha_{+}^{p}, \\ 0 & -\alpha_{+}^{p}<k_{y}<\alpha_{+}^{p}, \\ -2 & \alpha_{+}^{p}<k_{y} \leq \pi,\end{cases}
$$

for $0<\mu<4 t$ with $\alpha_{ \pm}^{p}=\arccos (-\mu / 2 t \pm 1)$.

Another topological number $\left(\mathbf{Z}_{2}\right.$ topological number [82]) can be defined for the spin-triplet $p_{x}$-wave superconductor in the presence of the RSOC for $k_{y}=0$ or $\pi$. At $k_{y}=0$ or $\pi$, the Hamiltonian given by Eq. (18) possesses time-reversal symmetry $\left(T=i \sigma_{2} \tau_{0} K\right)$ and particle-hole symmetry $\left(C=i \sigma_{0} \tau_{1} K\right)$ :

$$
\begin{aligned}
& T^{-1} H_{p}\left(k_{x}, k_{y}=\eta\right) T=H_{p}\left(-k_{x}, k_{y}=\eta\right), \\
& C^{-1} H_{p}\left(k_{x}, k_{y}=\eta\right) C=-H_{p}\left(-k_{x}, k_{y}=\eta\right),
\end{aligned}
$$

with $\eta=0$ or $\pi$. The eigenvectors of this Hamiltonian has a relation called Kramers degeneracy, i.e.,

$$
\begin{gathered}
\left|u_{\alpha}^{I}\left(-k_{x}, k_{y}=\eta\right)\right\rangle=-e^{i \chi_{\alpha, \eta}\left(k_{x}\right)} T\left|u_{\alpha}^{I I}\left(k_{x}, k_{y}=\eta\right)\right\rangle, \\
\left|u_{\alpha}^{I I}\left(-k_{x}, k_{y}=\eta\right)\right\rangle=e^{i \chi_{\alpha, \eta}\left(-k_{x}\right)} T\left|u_{\alpha}^{I}\left(k_{x}, k_{y}=\eta\right)\right\rangle,
\end{gathered}
$$


where $I$ and $I I$ indicate the Kramers degeneracy index, $\alpha$ denotes the band index and $\chi_{\alpha, \eta}\left(k_{x}\right)$ is a $U(1)$ phase factor. The Hamiltonian given by Eq. (18) with $k_{y}=0$ or $\pi$ belongs to DIII class in one dimension and it is characterized by $\mathbf{Z}_{2}$ topological number. $\mathbf{Z}_{2}$ topological number is defined by the one of the Kramers pair as

$$
(-1)^{\nu_{\eta}^{I}}=(-1)^{\nu_{\eta}^{I I}}= \pm 1
$$

with

$$
\begin{aligned}
\nu_{\eta}^{\beta} & =\exp \left[i \int_{0}^{2 \pi} d k_{x} A_{\eta}^{\beta}\left(k_{x}\right)\right] \\
A_{\eta}^{\beta}\left(k_{x}\right) & =-i \sum_{\alpha: \text { occ }}\left\langle u_{\alpha}^{\beta}\left(k_{x}, k_{y}=\eta\right)\left|\partial_{k_{x}}\right| u_{\alpha}^{\beta}\left(k_{x}, k_{y}=\eta\right)\right\rangle .
\end{aligned}
$$

Here $\beta=I$ or $I I$, summation in Eq. (35) runs over its occupied bands and $A_{\eta}^{\beta}\left(k_{x}\right)$ is a Berry connection. The system is topologically trivial when $(-1)^{\nu_{\eta}^{I}}=1$ and is topologically nontrivial when $(-1)^{\nu_{\eta}^{I}}=-1$. When $(-1)^{\nu_{\eta}^{I}}$ is -1 , there are ZESABSs. $\mathbf{Z}_{2}$ topological number for the spin-triplet $p_{x}$-wave superconductor with the RSOC is

$$
(-1)^{\nu_{\eta=0}^{I}}= \begin{cases}1 & \mu<-4 t \text { or } 0<\mu, \\ -1 & -4 t<\mu<0,\end{cases}
$$

for $k_{y}=0$ and it is

$$
(-1)^{\nu_{\eta=\pi}^{I}}= \begin{cases}1 & \mu<0 \text { or } 4 t<\mu, \\ -1 & 0<\mu<4 t\end{cases}
$$

for $k_{y}=\pi$.

\section{Spin-singlet $d_{x y}$-wave superconductor}

For the spin-singlet $d_{x y}$-wave case, ZESABSs can be understood by only using the winding number. The chiral operator is given by $\Gamma=\Gamma_{d} \equiv C T=-\sigma_{2} \tau_{1}$, and $\Gamma_{d}$ anticommutes with the Hamiltonian:

$$
\left\{H_{t}(\mathbf{k}), \Gamma_{d}\right\}=\left\{H_{\Delta}^{d}(\mathbf{k}), \Gamma_{d}\right\}=\left\{H_{\mathrm{SO}}(\mathbf{k}), \Gamma_{d}\right\}=0 .
$$

Then, the ZESABSs for the spin-singlet $d_{x y}$-wave superconductor are robust against the RSOC. The winding number for the spin-singlet $d_{x y}$-wave superconductor with the RSOC is complicated but for the parameters we choose in this paper $\left(\lambda / \Delta_{0} \leq 10\right.$ with $\Delta_{0} / t=0.01$ and $\mu / t=-2.4)$, the winding number is

$$
W\left(k_{y}\right)= \begin{cases}0 & k_{y}<-\alpha_{-}^{d}, \\ -1 & -\alpha_{-}^{d}<k_{y}<-\alpha_{+}^{d}, \\ -2 & -\alpha_{+}^{d}<k_{y}<0, \\ 2 & 0<k_{y}<\alpha_{+}^{d}, \\ 1 & \alpha_{+}^{d}<k_{y}<\alpha_{-}^{d}, \\ 0 & \alpha_{-}^{d}<k_{y}\end{cases}
$$

with

$$
\cos \alpha_{ \pm}^{d}=\frac{1}{4 t^{2}+\lambda^{2}}\left[-2 t(2 t+\mu) \pm \lambda \sqrt{\lambda^{2}-4 t \mu-\mu^{2}}\right] .
$$

Other cases with different parameters are shown in the Appendix B

\section{Results}

We discuss the momentum resolved surface DOS, the surface DOS and the odd-frequency spin-triplet $s$-wave pair amplitude at the surface for the spin-triplet $p_{x^{-} \text {wave }}$ and the spin-singlet $d_{x y}$-wave superconductors.

\section{Spin-triplet $p_{x}$-wave superconductor}

At first, we discuss the surface DOS for the spin-triplet $p_{x}$-wave superconductor. In Fig. 3, we show the momentum resolved surface DOS $\rho\left(E, k_{y}\right)$ given by Eq. (10) [Figs. 3 (a) to (d)] and the surface DOS $\bar{\rho}(E)$ given by Eq. (11) [Figs. 3 (e) to (h)]. As mentioned in Sect. IIC, without the RSOC, zero-energy surface Andreev bound states (ZESABSs) appear as a flat band edge state for $-\alpha_{-}^{p}<k_{y}<\alpha_{-}^{p}$ with $\alpha_{-}^{p}=\arccos (-\mu / 2 t-1)$ [Fig. 3 (a)] due to the non-zero value of the winding number. $\bar{\rho}(E)$ also exhibits sharp zero-energy peak (ZEP) as shown in Fig. 3 (e). Since ZESABSs for the spin-triplet $p_{x}$-wave superconductor are fragile against the RSOC [17, 81] (see Sect. IIC), ZESABSs split into two with the increase of the RSOC other than $k_{y}=0$ [Figs. 3 (b) and (f)]. As explained in Sect. IIC, at $k_{y}=0, \mathbf{Z}_{2}$ topological number has a nontrivial value when $-4 t<\mu<0$ and the ZESABS at $k_{y}=0$ is topologically protected provided that the bulk superconducting gap opens $\left(\lambda<\Delta_{0}\right)$. At $\lambda=\Delta_{0}$, the bulk gap closes [Eq. (8) and Figs. 3 (c) and (d)] and for $\lambda \gtrsim \Delta$, the corresponding $\bar{\rho}(E)$ is almost independent of $E$ [Figs. $3(\mathrm{~g})$ and (h)].

Next we discuss the odd-frequency spin-triplet $s$-wave pair amplitude at the surface $\left[P_{S_{z}}^{\text {triplet }}\left(\omega_{n}\right)\right.$ given in Eqs. (12) to (14)] shown in Fig. [4. As can be seen in these graphs, $P_{S_{z}}^{\text {triplet }}\left(\omega_{n}\right)$ is odd in $\omega_{n}$ due to the FermiDirac statistics. It is also noted that $P_{S_{z}}^{\text {triplet }}\left(\omega_{n}\right)$ satisfies $P_{S_{z}=1}^{\text {triplet }}\left(\omega_{n}\right)+P_{S_{z}=-1}^{\text {triplet }}\left(\omega_{n}\right)=0$ within numerical accuracy. For the spin-triplet $p_{x}$-wave superconductor without the RSOC [Fig. 团 (a)], $P_{S_{z}=0}^{\text {triplet }}\left(\omega_{n}\right)$ has a large value due to the translational symmetry breaking and $P_{S_{z}= \pm 1}^{\text {triplet }}\left(\omega_{n}\right)$ is zero due to the spin-rotational symmetry. $P_{S_{z}=0}^{\text {triplet }}\left(\omega_{n}\right)$ drastically decreases with the increase of $\lambda$ [Figs. 4 (b)] since the zero-energy flat band disappears other than $k_{y}=0$. On the other hand, divergent behavior very close to $\omega_{n}=0$ remains due to ZESABS at $k_{y}=0$. In Figs. 15 (a) to (d) in the Appendix C] we also show the odd-frequency $s$-wave pair amplitude at the surface with $\lambda / \Delta_{0}=0,0.1,1$ and 10 . 


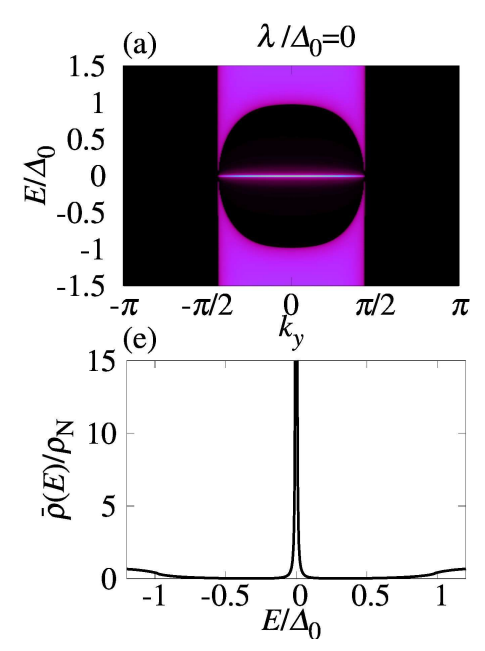

semi-infinite spin-triplet $p_{x}$-wave superconductor
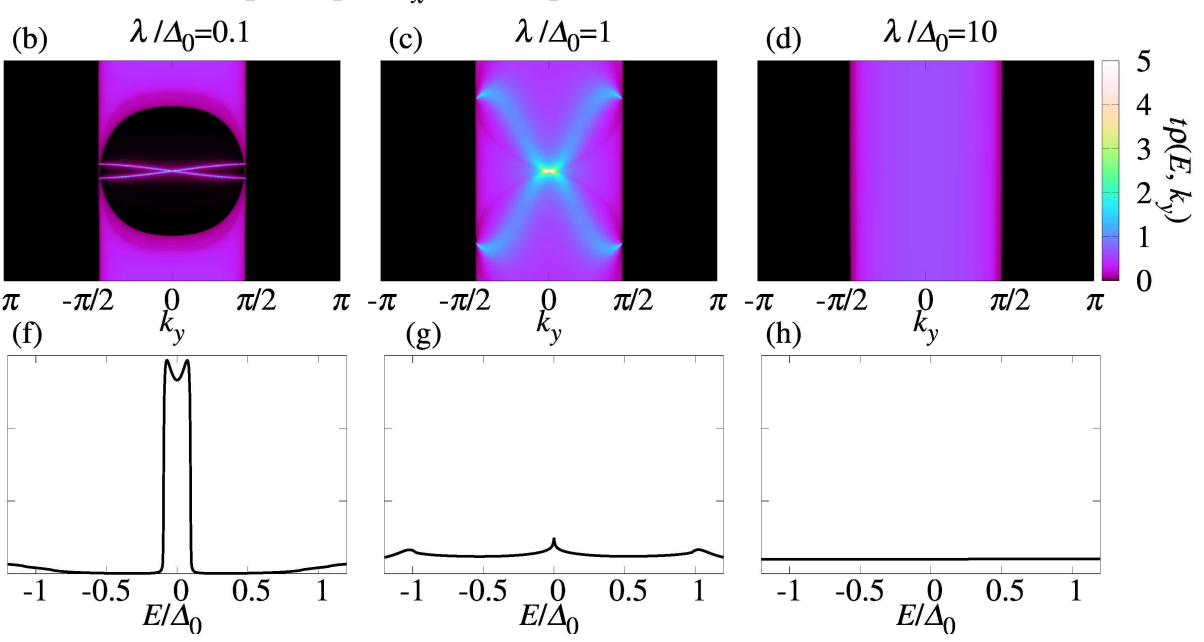

FIG. 3. The momentum resolved surface DOS $\left[\rho\left(E, k_{y}\right)\right.$ from (a) to (d)] and the surface DOS $[\bar{\rho}(E)$ from (e) to (h)] for the spin-triplet $p_{x}$-wave superconductor are plotted for several $\lambda / \Delta_{0}$. Here $\rho_{\mathrm{N}}$ is a surface density of states at zero-energy with the normal state $\left(\Delta_{0}=0\right) . \rho\left(E, k_{y}\right)$ and $\bar{\rho}(E)$ are calculated at $j_{x}=1$ (surface) in the system shown in Fig. 2 The figures in the same column have the same $\lambda$. [(a), (e)] $\lambda=0,[(\mathrm{~b}),(\mathrm{f})] \lambda / \Delta_{0}=0.1,[(\mathrm{c}),(\mathrm{g})] \lambda / \Delta_{0}=1$, and $[(\mathrm{d}),(\mathrm{h})] \lambda / \Delta_{0}=10$ with $\Delta_{0} / t=0.01$.

spin-triplet $p_{x}$-wave superconductor

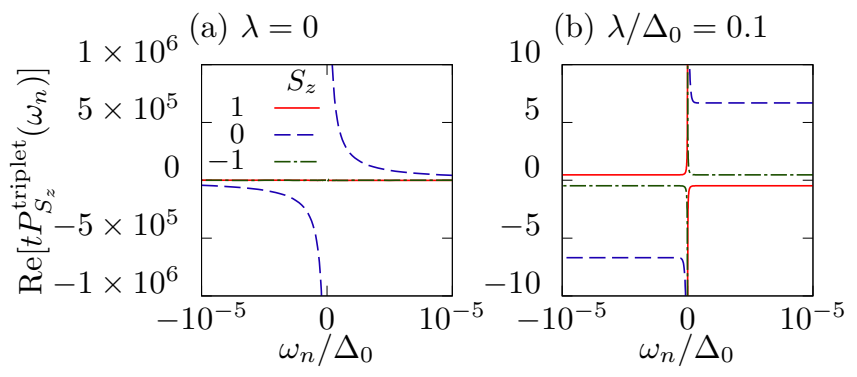

FIG. 4. The real part of the odd-frequency spin-triplet $s$ wave pair amplitude $P_{S_{z}}^{\text {triplet }}\left(\omega_{n}\right)$ at the surface is plotted as a function of $\omega_{n} / \Delta_{0}$ for $S_{z}=1,0,-1$ for the spin-triplet $p_{x}$-wave superconductor. (a) $\lambda / \Delta_{0}=0$ and (b) $\lambda / \Delta_{0}=0.1$. The imaginary part of $P_{S_{z}}^{\text {triplet }}\left(\omega_{n}\right)$ is zero within numerical accuracy.

The total odd-frequency spin-triplet $s$-wave pair amplitude at the surface $P^{\text {triplet }}\left(\omega_{n}\right)$ given by Eq. (15) with $\omega_{n} / \Delta_{0}=10^{-3}$ is shown in Fig. [5] For the spin-triplet $p_{x}$-wave case, $P^{\text {triplet }}\left(\omega_{n}\right)$ has a large value at $\lambda=0$. On the other hand, with the non-zero value of the RSOC, $P^{\text {triplet }}\left(\omega_{n}\right)$ decreases drastically due to the absence of the ZESABS other than $k_{y}=0 . P^{\text {triplet }}\left(\omega_{n}\right)$ has a peak approximately at $\lambda / \Delta_{0}=1$ where the bulk energy gap closes. The surface DOS at zero-energy also exhibits a similar behavior [In the inset of Fig. 5] since the slope of the dispersive surface Andreev bound state at $k_{y}=0$ becomes smaller as $\lambda / \Delta_{0}=1-\delta$ approaches unity from $\delta \rightarrow+0$.

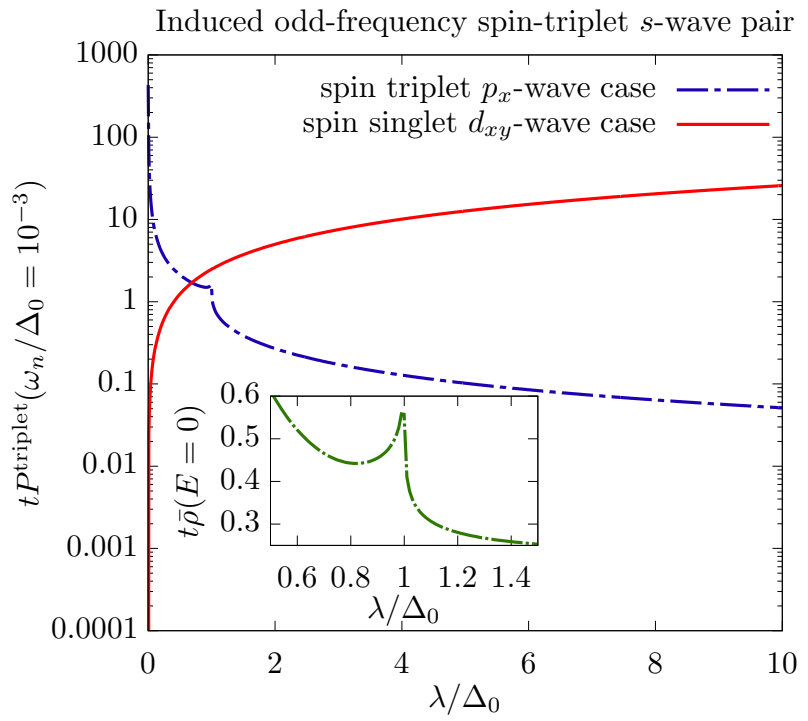

FIG. 5. The odd-frequency spin-triplet $s$-wave pair amplitude $P^{\text {triplet }}\left(\omega_{n} / \Delta_{0}=10^{-3}\right)$ given by Eq. (15) is plotted as a function of the RSOC $\lambda$ for the spin-triplet $p_{x}$-wave superconductor and the spin-singlet $d_{x y}$-wave superconductor. In the inset, the surface $\operatorname{DOS} \bar{\rho}(E=0)$ is plotted as a function of $\lambda$. Here $\Delta_{0} / t=0.01$.

\section{Spin-singlet $d_{x y}$-wave superconductor}

In Fig. 6. $\rho\left(E, k_{y}\right)$ and $\bar{\rho}(E)$ for the spin-singlet $d_{x y}$-wave superconductor are shown. As explained in Sect. IIC] ZESABSs for the spin-singlet $d_{x y}$-wave superconductor are robust against the RSOC shown in Figs. 6 (a) to (d). The regime for the ZESABSs are 

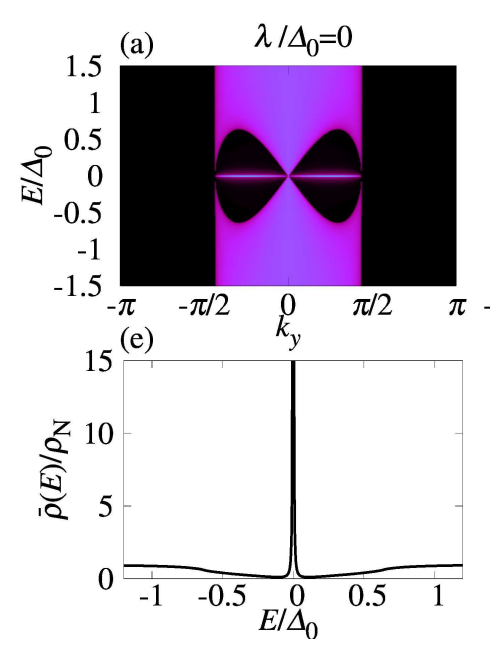

semi-infinite spin-singlet $d_{x y}$-wave superconductor
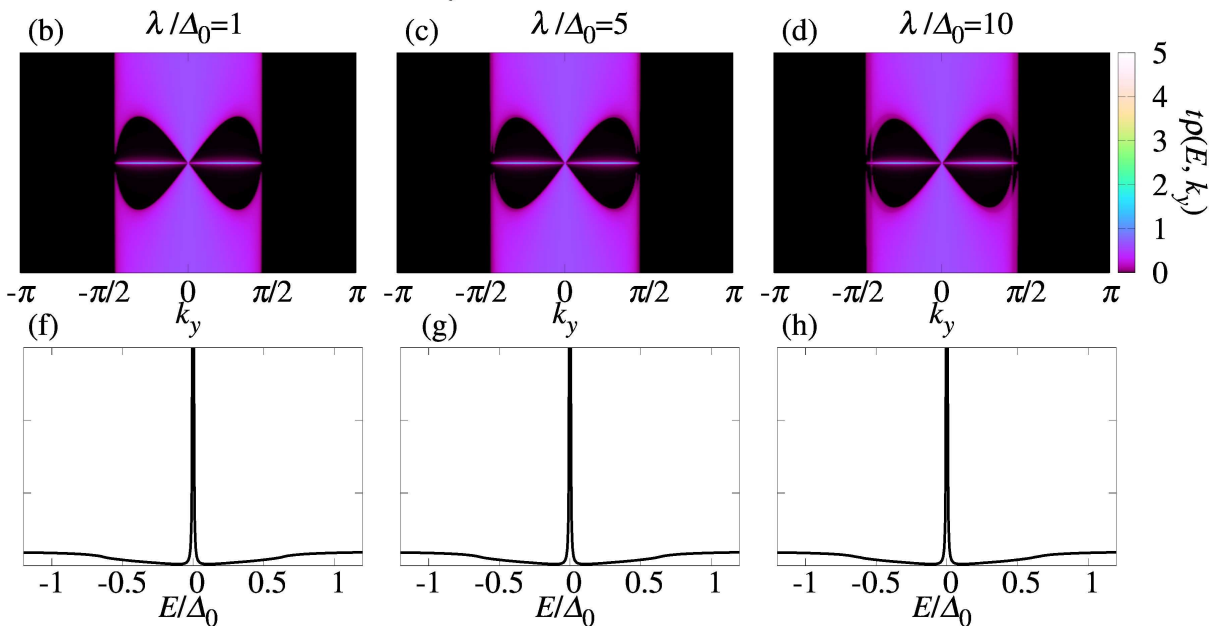

FIG. 6. The momentum resolved surface DOS $\left[\rho\left(E, k_{y}\right)\right.$ from (a) to (d)] and the surface DOS $[\bar{\rho}(E)$ from (e) to (h)] for the spin-singlet $d_{x y}$-wave superconductor are plotted for several $\lambda / \Delta_{0}$. Here $\rho_{\mathrm{N}}$ is a surface density of states at zero-energy with the normal state $\left(\Delta_{0}=0\right) . \rho\left(E, k_{y}\right)$ and $\bar{\rho}(E)$ are calculated at $j_{x}=1$ (surface) in the system shown in Fig. 2 . The figures in the same column have the same $\lambda$. [(a), (e)] $\lambda=0,[(\mathrm{~b}),(\mathrm{f})] \lambda / \Delta_{0}=1,[(\mathrm{c}),(\mathrm{g})] \lambda / \Delta_{0}=5$, and $[(\mathrm{d}),(\mathrm{h})] \lambda / \Delta_{0}=10$ with $\Delta_{0} / t=0.01$.

given by Eq. (39). From Eq. (9), for a non-zero value of $\lambda$, degeneracy of the point nodes is lifted. The splitting of the point nodes is too small to recognize in Figs. 6 (b) and (c), but for $\lambda / \Delta_{0}=10$, it can be seen in Fig. 6 (d). The surface DOS is almost independent of the strength of the RSOC as shown in Figs. 6 (e) to (h) due to the existence of the ZESABSs.

The odd-frequency spin-triplet $s$-wave pair amplitude is absent for $\lambda=0$ due to the spin-rotational symmetry. It is noted that the odd-frequency spin-singlet $p_{y^{-}}$ wave pair amplitude has a large value [Fig. 16 (a) in the Appendix D. On the other hand, $P_{S_{z}}^{\text {triplet }}\left(\omega_{n}\right)$ increases with the increase of $\lambda$ [Fig. 7 and Figs. 15] (e) to (f) in the Appendix [C]. The amplitude of the $P_{S_{z}= \pm 1}^{\text {triplet }}\left(\omega_{n}\right)$ is larger than that of the $P_{S_{z}=0}^{\text {triplet }}\left(\omega_{n}\right)$ for $\lambda>0$ since the $S_{z}=0$ spin-triplet component of the anomalous Green's function in the bulk system is zero [Appendix E].

The total odd-frequency spin-triplet $s$-wave pair amplitude at the surface $P^{\text {triplet }}\left(\omega_{n}\right)$ given by Eq. (15). $P^{\text {triplet }}\left(\omega_{n}\right)$ is zero at $\lambda=0$ due to the spin-rotational symmetry and it becomes larger with the increase of $\lambda$. From this result, we can expect that the anomalous proximity effect occurs for the spin-singlet $d_{x y}$-wave case with a large value of the RSOC.

\section{DIFFUSIVE NORMAL METAL/SUPERCONDUCTOR JUNCTION}

In this section, we discuss the local DOS and the oddfrequency spin-triplet $s$-wave pair amplitude in the diffusive normal metal (DN) for DN/SC junctions with the RSOC (shown in Fig. 8). spin-singlet $d_{x y}$-wave superconductor

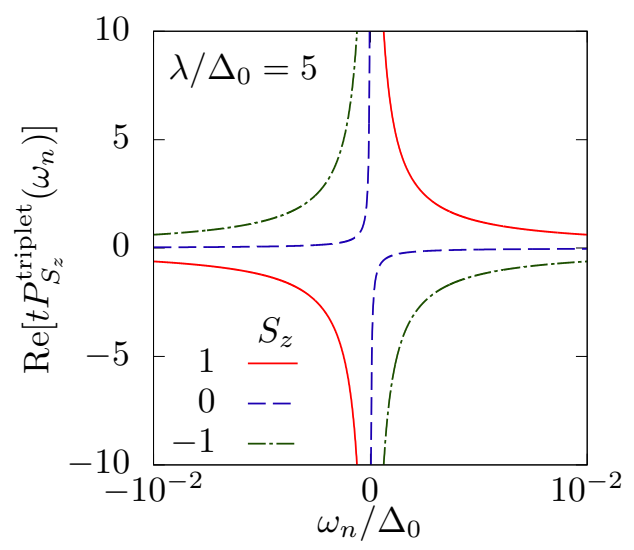

FIG. 7. The real part of the odd-frequency spin-triplet $s$ wave pair amplitude $P_{S_{z}}^{\text {triplet }}\left(\omega_{n}\right)$ at the surface is plotted as a function of $\omega_{n} / \Delta_{0}$ for $S_{z}=1,0,-1$ for the spin-singlet $d_{x y^{-}}$ wave superconductor with $\lambda / \Delta_{0}=5$. The imaginary part of $P_{S_{z}}^{\text {triplet }}\left(\omega_{n}\right)$ is zero within numerical accuracy.

\section{A. Model and Method}

The Hamiltonian for the SC $\left(j_{x} \geq 1\right)$ is given by Eq. (1) and the Hamiltonian for the DN $\left(-49 \leq j_{x} \leq 0\right)$ is

$$
\begin{aligned}
& \mathcal{H}_{\mathrm{DN}}^{l_{s}} \\
& =-t \sum_{\langle\mathbf{i}, \mathbf{j}\rangle, \sigma}\left(c_{\mathbf{i}, \sigma}^{\dagger} c_{\mathbf{j}, \sigma}+\text { H.c. }\right)+\sum_{-49 \leq j_{x} \leq 0, j_{y}}\left(-\mu+V_{\mathbf{j}}^{l_{s}}\right) n_{\mathbf{j}},
\end{aligned}
$$




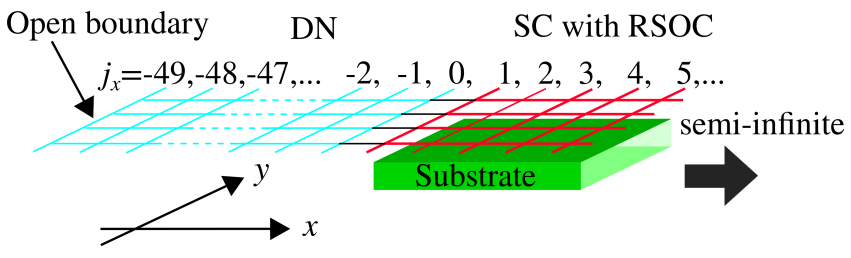

FIG. 8. Schematic illustration of DN without RSOC $(-49 \leq$ $\left.j_{x} \leq 0\right) /$ semi-infinite $\left(j_{x} \geq 1\right)$ SC with RSOC junction.

where the summations in the $x$-direction run over from -49 to $0\left(-49 \leq i_{x}, j_{x} \leq 0\right)$, and those in the $y$-direction run over from 1 to $L_{y}\left(1 \leq i_{y}, j_{y} \leq L_{y}\right) . V_{\mathbf{j}}^{l_{s}}\left(-t \leq V_{\mathbf{j}}^{l_{s}} \leq\right.$ $t$ with $\left.l_{s}=1,2, \ldots, N_{\text {sample }}\right)$ is a randomly chosen onsite impurity potential in the DN and there is no RSOC in the DN. The Hamiltonian which connects the DN and the $\mathrm{SC}$ is

$$
\mathcal{H}_{\text {connect }}=-t \sum_{j_{y}, \sigma}\left(c_{\left(0, j_{y}\right), \sigma}^{\dagger} c_{\left(1, j_{y}\right), \sigma}+\text { H.c. }\right) \text {. }
$$

The local Green's function is obtained from the same procedure explained in Sect. IIB. The LDOS in the DN is given by

$$
\begin{aligned}
\bar{\rho}\left(E, j_{x}\right) & =-\frac{1}{2 N_{k_{y}} L_{y} N_{\text {sample }}} \\
& \times \sum_{j_{y}=j_{y}^{\prime}, k_{y}, l_{s}} \frac{1}{\pi} \operatorname{Im}\left[\operatorname{tr} \tilde{g}_{j_{x}}^{\mathrm{DN}, l_{s}}\left(z=E+i \eta, j_{y}, j_{y}^{\prime}, k_{y}\right)\right],
\end{aligned}
$$

where we use a super unit cell $k_{y}=\pi m /\left(N_{k_{y}} L_{y}\right)$ with $m=-N_{k_{y}}+1, \ldots, N_{k_{y}}, N_{k_{y}}=10$ and $L_{y}$ is a length of the super unit cell in the $y$-direction used in numerical calculation. Here, trace is only taken in particle space and we set $\eta / t=10^{-5}$. The Green's function in the DN $\tilde{g}_{j_{x}}^{\mathrm{DN}, l_{s}}\left(z, j_{y}, j_{y}^{\prime}, k_{y}\right)$ in Eq. (43) is given in the Appendix $[\mathrm{F}$ [Eq. (F12)]. To calculate the LDOS for the spin-triplet $p_{x}$-wave superconductor junction, we take $L_{y}=120$ which is the length of the system in the $y$ direction and to reduce the system size effect, 60 samples for $V_{j}^{l_{s}}\left(l_{s}=1,2, \ldots, 60\right.$, i.e., $\left.N_{\text {sample }}=60\right)$ are used to calculate the averaged value of the LDOS and the oddfrequency pair amplitude in the DN. We take $L_{y}=240$ and 12 samples $\left(N_{\text {sample }}=12\right)$ are used to the impurity average for the spin-singlet $d_{x y}$-wave junction since size effect for the spin-singlet $d_{x y}$-wave case is larger than that for the spin-triplet $p_{x}$-wave case since the pair potential for the spin-singlet $d_{x y}$-wave superconductor depends on $k_{y}$ but that for the spin-triplet $p_{x}$-wave superconductor does not depend on $k_{y}$.

To discuss the relation between the anomalous proximity effect and the odd-frequency spin-triplet $s$-wave pair amplitude, we calculate its averaged value in the DN re- gion given by

$$
\begin{aligned}
& \bar{P}_{S_{z}=1}^{\text {DN,triplet }}\left(\omega_{n}\right)=\frac{1}{N} \sum_{j_{x}=N_{\mathrm{i}}}^{N_{\mathrm{f}}} P_{j_{x}, \uparrow, \uparrow}\left(\omega_{n}\right), \\
& \bar{P}_{S_{z}=0}^{\text {DN,triplet }}\left(\omega_{n}\right)=\frac{1}{\sqrt{2} N} \sum_{j_{x}=N_{\mathrm{i}}}^{N_{\mathrm{f}}}\left[P_{j_{x}, \uparrow, \downarrow}\left(\omega_{n}\right)+P_{j_{x}, \downarrow, \uparrow}\left(\omega_{n}\right)\right], \\
& \bar{P}_{S_{z}=-1}^{\text {DN,triplet }}\left(\omega_{n}\right)=\frac{1}{N} \sum_{j_{x}=N_{\mathrm{i}}}^{N_{\mathrm{f}}} P_{j_{x}, \downarrow, \downarrow}\left(\omega_{n}\right),
\end{aligned}
$$

with

$$
\begin{aligned}
& \frac{P_{j_{x}, \sigma, \sigma^{\prime}}\left(\omega_{n}\right)}{2 N_{k_{y}} L_{y} N_{\text {sample }}} \sum_{k_{y}, j_{y}=j_{y}^{\prime}, l_{s}} \hat{f}_{j_{x}, \sigma, \sigma^{\prime}}^{l_{s}}\left(i \omega_{n}, j_{y}, j_{y}^{\prime}, k_{y}\right),
\end{aligned}
$$

where we average the odd-frequency pair amplitude in the DN: $N_{\mathrm{i}}=-49, N_{\mathrm{f}}=0$ and $N=N_{\mathrm{f}}-N_{\mathrm{i}}+1=50$. Here $2 L_{y} \times 2 L_{y}$ matrix $\hat{f}_{j_{x}, \sigma, \sigma^{\prime}}^{l_{s}}\left(z=i \omega_{n}, j_{y}, j_{y}^{\prime}, k_{y}\right)$ is an anomalous Green's function of $\tilde{g}_{j_{x}}^{\mathrm{DN}, l_{s}}\left(z, j_{y}, j_{y}^{\prime}, k_{y}\right)$ in Eq. (43) defined as the same manner as in Eq. (17). To calculate the odd-frequency spin-triplet $s$-wave pair amplitude, we take the same system size in the $y$-direction and the same number of the samples are used to the impurity average.

\section{B. Results}

We discuss the LDOS and the odd-frequency spintriplet $s$-wave pair amplitude in the $\mathrm{DN}$ for the spintriplet $p_{x}$-wave and the spin-singlet $d_{x y}$-wave superconductor junction.

\section{Spin-triplet $p_{x}$-wave superconductor junction}

Firstly, we discuss the LDOS in the DN corresponding to Fig. 8. LDOS for the spin-triplet $p_{x}$-wave superconductor junction without the RSOC has a sharp ZEP as shown in Fig. 9 (a) consistent with the previous results in quasiclassical regime [26]. It is called anomalous proximity effect $[26-28]$. The ZEP on LDOS in the DN stems from ZESABSs as a flat band zero-energy state in semiinfinite spin-triplet $p_{x}$-wave superconductor. The present ZESABS accompanies OTE $s$-wave pairing, then it can penetrate into the DN. However, after switching on $\lambda$ in the spin-triplet $p_{x}$-wave superconductor, the ZEP of the LDOS in the DN is suppressed by $\lambda$, as shown in Fig. 9 (b). In this case, if we look at the semi-infinite spin-triplet $p_{x}$-wave superconductor without the $\mathrm{DN}$, the zero-energy flat bands at the surface split into two [Figs. 3 (b) and (f)] and the surface DOS at zero-energy is suppressed. This is the reason why the LDOS at zero-energy 


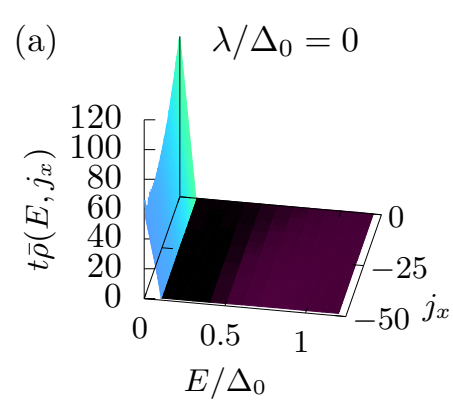

spin-triplet $p_{x}$-wave superconductor junction
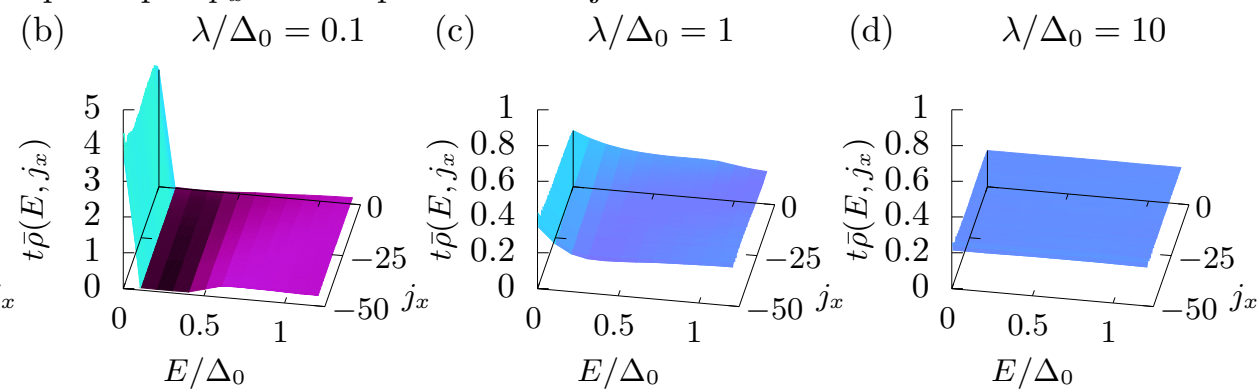

FIG. 9. The LDOS $\left[\bar{\rho}\left(E, j_{x}\right)\right]$ in the DN is plotted as functions of $j_{x}$ and $E / \Delta_{0}$ for several strength of the RSOC for the spin-triplet $p_{x}$-wave superconductor junction. The amplitude of the RSOC is (a) $\lambda / \Delta_{0}=0$, (b) 0.1 , (c) 1 , and (d) 10 with $\Delta_{0} / t=0.01$.
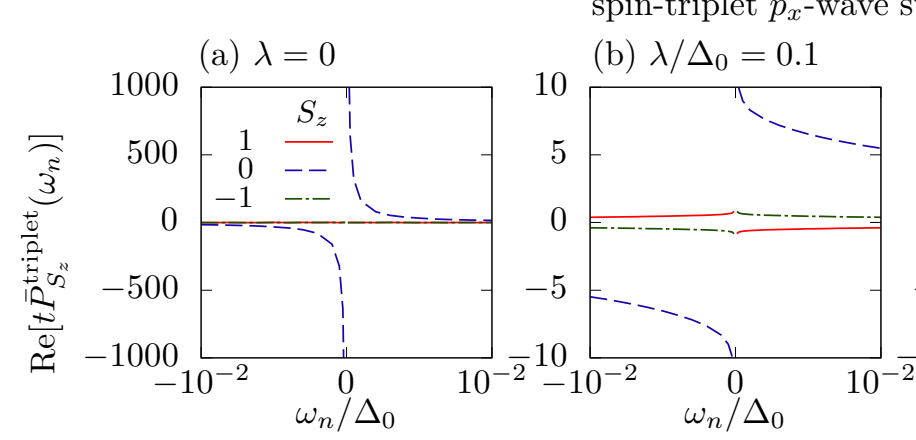

spin-triplet $p_{x}$-wave superconductor junction

(b) $\lambda / \Delta_{0}=0.1$

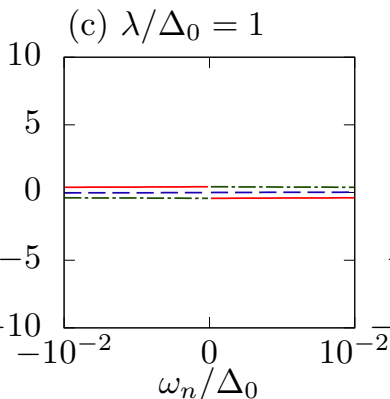

(d) $\lambda / \Delta_{0}=10$

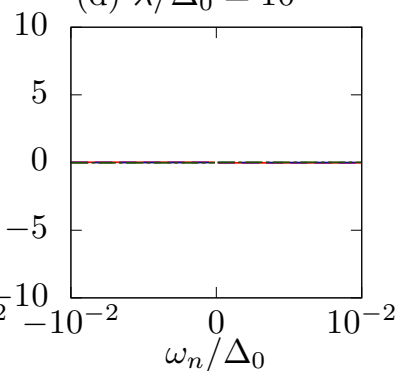

FIG. 10. The averaged value of the real part of the odd-frequency spin-triplet $s$-wave pair amplitude in the DN $\bar{P}_{S_{z}}^{\text {triplet }}\left(\omega_{n}\right)$ is plotted as a function of $\omega_{n}$ for the spin-triplet $p_{x}$-wave superconductor junction with $S_{z}=1,0$ and -1 . The imaginary part of $\bar{P}_{S_{z}}^{\text {triplet }}\left(\omega_{n}\right)$ is zero within numerical accuracy. (a) $\lambda / \Delta_{0}=0$, (b) 0.1 , (c) 1 , and (d) 10 .

in the $\mathrm{DN}$ is suppressed in the $\mathrm{DN} /$ spin-triplet $p_{x}$-wave superconductor junction in the presence of the RSOC. For $\lambda$ larger than $\Delta_{0}$, the bulk energy gap closes [see Figs. [3 (c) and (d)] and the ZEP almost vanishes [Figs. 9 (c) and (d)].

Corresponding to the suppression of the height of ZEP in the DN, the averaged value of the odd-frequency spintriplet $s$-wave pair amplitude $\bar{P}_{S_{z}=0}^{\mathrm{DN}, \text { triplet }}\left(\omega_{n}\right)$ given by Eq. (45) in the DN becomes smaller with the increase of the RSOC [Figs. 10 (a) and (b)] and this behavior of $\bar{P}_{S_{z}=0}^{\mathrm{DN} \text {,triplet }}\left(\omega_{n}\right)$ is similar to that for the semi-infinite system [Figs. 4 (a) and (b)]. The odd-frequency spintriplet $s$-wave pair amplitude $\bar{P}_{S_{z}= \pm 1}^{\mathrm{DN} \text {,triplet }}\left(\omega_{n}\right)$ is zero for $\lambda=0$ due to the spin-rotational symmetry and it has non-zero value for $\lambda>0$ [Figs. 10 (b) and (c)] and it also satisfies $\bar{P}_{S_{z}=1}^{\text {DN,triplet }}\left(\omega_{n}\right)+\bar{P}_{S_{z}=-1}^{\text {DN,triplet }}\left(\omega_{n}\right)=0$ within numerical accuracy. For large magnitude of $\lambda$, all kinds of $\bar{P}_{S_{z}}^{\text {DN,triplet }}\left(\omega_{n}\right)$ becomes almost zero [Fig. 10 (d)]. This result is similar to that for semi-infinite system shown in Fig. 4 and Figs. 15 (a) to (d) in the Appendix C]

\section{Spin-singlet $d_{x y}$-wave superconductor junction}

For the spin-singlet $d_{x y}$-wave superconductor without the RSOC, the LDOS in the DN is nearly constant as a function of $E$ and $j_{x}$ [Fig. 11 (a)]. It is known from the quasiclassical theory of proximity effect in unconventional superconductor, that the LDOS is reduced to that in the normal state due to the absence of the proximity effect in the DN [83, 84]. The slight energy dependence is due to the finite size effect in the numerical calculation of the lattice model. A similar behavior also appears for $\lambda / \Delta_{0}=1$ [Fig. 11](b)]. However, for a large RSOC values $\left(\lambda / \Delta_{0}=5\right.$ and 10), the LDOS has a ZEP in the DN region [Figs.11](c) and (d)]. In Fig. 12, we show the LDOS at the center of the DN $\left(j_{x}=-25\right)$ and for $\lambda / \Delta_{0} \gtrsim 2$, the ZEP appears. Therefore, it may be possible to detect the anomalous proximity effect for the spin-singlet $d$-wave superconductor if the amplitude of the RSOC becomes larger than that of the pair potential. System size and the number of samples dependence of the LDOS are discussed in the Appendix G.

At $\lambda=0$, the odd-frequency spin-triplet $s$-wave pair amplitude $\bar{P}_{S_{z}}^{\mathrm{DN} \text {,triplet }}\left(\omega_{n}\right)$ is zero due to the spinrotational symmetry [Figs. 13 (a)]. The amplitude of $\bar{P}_{S_{z}}^{\text {DN,triplet }}\left(\omega_{n}\right)$ increases as the increase of $\lambda$ as shown 


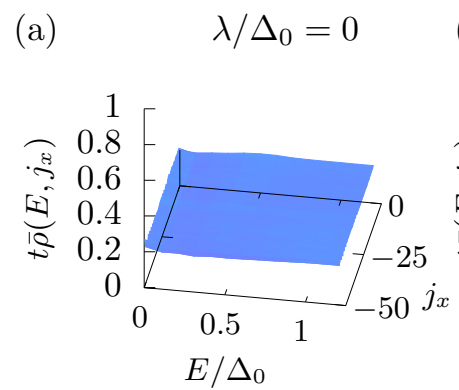

spin-singlet $d_{x y}$-wave superconductor junction
(b)
$\lambda / \Delta_{0}=1$
(c) $\quad \lambda / \Delta_{0}=5$

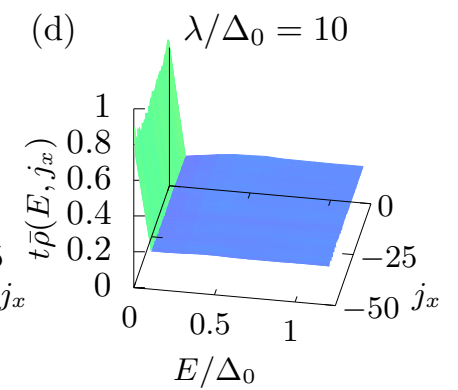

FIG. 11. The LDOS $\left[\bar{\rho}\left(E, j_{x}\right)\right]$ in the DN is plotted as functions of $j_{x}$ and $E / \Delta_{0}$ for several strength of the RSOC for the spin-singlet $d_{x y}$-wave superconductor junction. The amplitude of the RSOC is (a) $\lambda / \Delta_{0}=0$, (b) 1 , (c) 5 , and (d) 10 with $\Delta_{0} / t=0.01$.

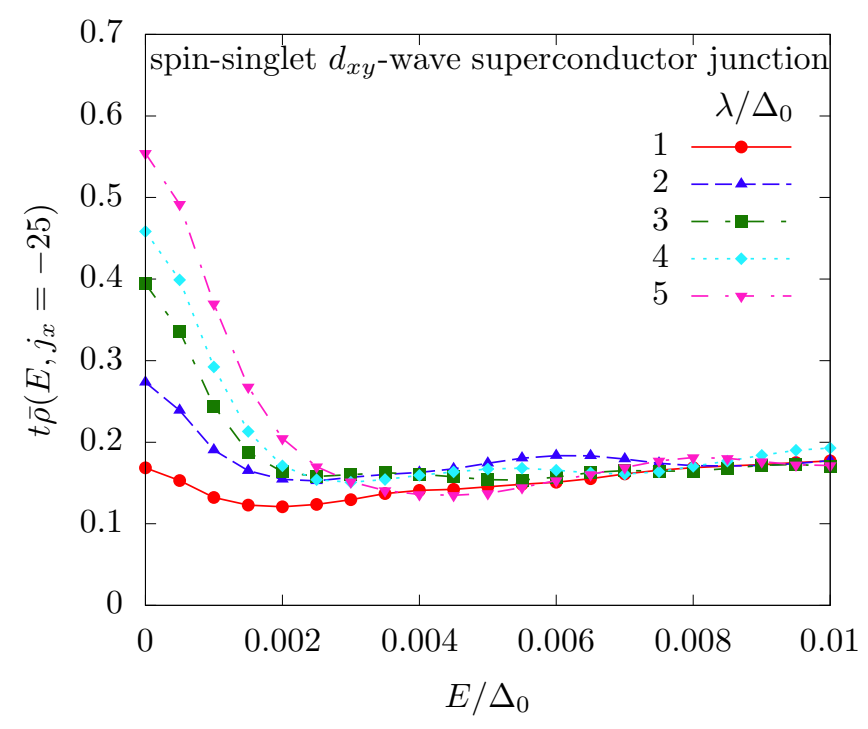

FIG. 12. The LDOS for the spin-singlet $d_{x y}$-wave superconductor junction at $j_{x}=-25$ (center of the $\mathrm{DN}$ ) is plotted as a function of $E / \Delta_{0}$ for several $\lambda / \Delta_{0}$ with $\Delta_{0} / t=0.01$.

in Figs. 13 (b) to (d). Here, non-zero value of the oddfrequency spin-triplet $s$-wave pair amplitude in the DN means that it is robust against the impurity scattering in the DN [spatial dependence is shown in Fig. 20 in the Appendix [H]. Qualitative behavior of the oddfrequency spin-triplet $s$-wave pair amplitude in the DN as a function of the RSOC [Figs. 13 (a) to (d)] is the same as that for the semi-infinite $\mathrm{SC}$ system at the surface i.e., it increases as the increase of the RSOC [see also Figs. 15 (e) to (h) in the Appendix [C. The oddfrequency spin-triplet $s$-wave pair amplitude also satisfies $\bar{P}_{S_{z}=1}^{\mathrm{DN}, \text { triplet }}\left(\omega_{n}\right)+\bar{P}_{S_{z}=-1}^{\mathrm{DN}, \text { triplet }}\left(\omega_{n}\right)=0$ within numerical accuracy. The odd-frequency spin-singlet $p_{y}$-wave pair amplitude rapidly decays in the DN since it is fragile against impurity scattering [Appendix $\mathrm{H}$.

\section{SUMMARY}

In this paper, we have studied the proximity effect in the $\mathrm{DN} /$ superconductor junctions, focusing on the anomalous proximity effect, where the LDOS in the DN has a zero-energy peak. For the spin-triplet $p_{x}$-wave superconductor case, when the direction of the $\mathbf{d}$-vector is parallel to the $z$-direction, ZESABSs are suppressed by the RSOC $\lambda$ and the anomalous proximity effect disappears for large magnitude of $\lambda$.

It should be also noted that the anomalous proximity effect is switched on by the RSOC in spin-singlet $d_{x y}$-wave superconductor junctions. The resulting zeroenergy LDOS and the magnitude of the odd-frequency spin-triplet $s$-wave pair amplitude is enhanced with the increase of the magnitude of $\lambda$. This indicates that high $T_{\mathrm{c}}$ cuprate junctions can be used to detect the anomalous proximity effect. Another candidate material is $\mathrm{CeCoIn}_{5}$, where the promising pairing symmetry is spin-singlet $d$ wave [85]. It has been reported that the amplitude of the RSOC exceeds that of the superconducting gap 86 in the superlattices of $\mathrm{CeCoIn}_{5}$.

We comment on the effect of surface roughness on the odd-frequency spin-triplet $s$-wave pair amplitude for the spin-singlet $d_{x y}$-wave superconductor. It is known that in the presence of SABSs, pair potential decays near the surface [87 90]. It has been clarified that the surface roughness influences on the SABS and the resulting height of ZEP is suppressed by roughness 91, 92 since the induced odd-frequency pairing near the interface has a odd-parity with $p_{y}$-wave like symmetry [93]. On the other hand, in the presence of the RSOC, we can generate odd-frequency spin-triplet $s$-wave pair amplitude near the interface. The present pair amplitude is robust against the surface diffusive scattering 35]. In order to clarify this point, we have calculated spin-triplet $s$-wave pair amplitude in diffusive layer attached to spin-singlet $d_{x y}$-wave superconductor junction with the RSOC in the presence of the spatial depletion of the pair potential near the surface [Appendix I]. We have checked the robustness of the odd-frequency spin-triplet $s$-wave pair amplitude against diffusive scattering. Even if the spatial depletion 
spin-singlet $d_{x y}$-wave superconductor junction
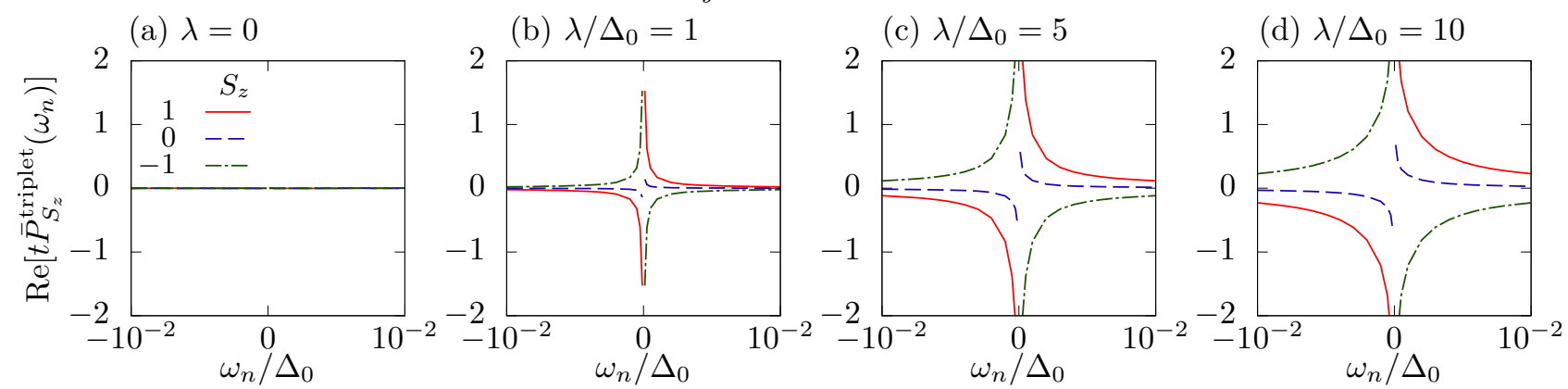

FIG. 13. The averaged value of the real part of the odd-frequency spin-triplet $s$-wave pair amplitude in the DN $\bar{P}_{S_{z}}^{\text {triplet }}\left(\omega_{n}\right)$ is plotted as a function of $\omega_{n}$ for the spin-singlet $d_{x y}$-wave superconductor junction with $S_{z}=1,0$ and -1 . The imaginary part of $\bar{P}_{S_{z}}^{\text {triplet }}\left(\omega_{n}\right)$ is zero within numerical accuracy. (a) $\lambda / \Delta_{0}=0$, (b) 1 , (c) 5 , and (d) 10 .

of the pair potential near the surface exists, the magnitude of the odd-frequency spin-triplet $s$-wave pair amplitude does not almost change as compared to the case with the constant pair potential up to the surface.

In this paper, we assume that a Rashba spin-orbit coupling exists in the two-dimensional superconductor uniformly along the $z$-direction, as superconductor is assumed to be thin. In addition to this effect, spin-orbit coupling also exists near the interface, on the plane parallel to the interface [94, 95]. In this case, the direction of the Rashba vector is different. For spin-singlet pair potentials, the direction of the spin-orbit coupling only affects the direction of the spin of the induced oddfrequency spin-triplet pair amplitude due to the spinrotational symmetry. On the other hand, for spin-triplet pair potentials, the relative angle of the $\mathbf{d}$-vector and the Rashba spin-orbit coupling affects surface Andreev bound states like diffusive ferromagnet/superconductor junctions [96]. It is also interesting to study this type of spin-orbit coupling.

We comment on the case that the amplitude of the RSOC is much larger than the hopping integral for the spin-singlet $d_{x y}$-wave superconductor. In our study, we consider that the amplitude of the RSOC is smaller than the hopping integral $t$ and the odd-frequency spin-triplet $s$-wave pair amplitude increases with the increase of the amplitude of the RSOC. On the other hand, when the amplitude of the RSOC is much larger than the hopping integral, the ZESABS disappears when the value of the chemical potential is non-zero as can be seen in the Appendix A and the odd-frequency spin-triplet $s$-wave pair amplitude disappears as increasing $\lambda / t$ for the large amplitude of the RSOC.

A ZESABS is also produced by a Dresselhaus spinorbit interaction and the Zeeman field [31]. The Dresselhaus spin-orbit interaction breaks the spin-rotational symmetry and the anomalous proximity effect is also expected for the spin-singlet $d_{x y}$-wave superconductors.

Recently, relation between crossed Andreev reflection (CAR) and odd frequency pair amplitude is pointed out [58]. On the other hand, in ballistic transport regime, CAR with mixed parity state is calculated [97]. It is an interesting future problem to calculate CAR in diffusive regime.

In this paper, we focus on the localized odd-frequency pairing accompanied by the ZESABS, which is generated by the symmetry breaking from the bulk conventional even-frequency pair potential. On the other hand, several studies have been pursuing the realization of a bulk odd-frequency superconductor so far 18, 98 115]. Although it is difficult to express the bulk oddfrequency superconducting states consistent with various conditions [116, 117], a recently proposed odd-frequency gap function in the two channel Kondo lattice model (TCKL) is a promising system [118]. The calculation of charge transport and Josephson effect has already started [119]. It is interesting to study proximity effects in the DN/TCKL system.

\section{ACKNOWLEDGMENTS}

Y. Asano, S. Kobayashi S. Nakosai are thanked for useful discussions. This work was supported by Grantsin-Aid from JSPS for Scientific Research on Innovative Areas Topological Materials Science (KAKENHI Grant Numbers JP15H05851, JP15H05853 and JP15K21717), for Scientific Research (B) (KAKENHI Grant Numbers JP15H03686 and JP18H01176), and Japan-RFBR JSPS Bilateral Joint Research Projects/Seminars.
[1] L. J. Buchholtz and G. Zwicknagl, Identification of $p$ wave superconductors, Phys. Rev. B 23, 5788 (1981).
[2] J. Hara and K. Nagai, A Polar State in a Slab as a Sol- 
uble Model of $p$-Wave Fermi Superfluid in Finite Geometry, Progress of Theoretical Physics 76, 1237 (1986).

[3] C.-R. Hu, Midgap surface states as a novel signature for $d_{x_{a}^{2}-x_{b}^{2}}$-wave superconductivity, Phys. Rev. Lett. 72, 1526 (1994)

[4] S. Kashiwaya and Y. Tanaka, Tunnelling effects on surface bound states in unconventional superconductors, Reports on Progress in Physics 63, 1641 (2000).

[5] T. Löfwander, V. S. Shumeiko, and G. Wendin, Andreev bound states in high-tcsuperconducting junctions, Superconductor Science and Technology 14, R53 (2001)

[6] Y. Tanaka and S. Kashiwaya, Theory of tunneling spectroscopy of $d$-wave superconductors, Phys. Rev. Lett. 74, 3451 (1995)

[7] M. Covington, M. Aprili, E. Paraoanu, L. H. Greene, F. Xu, J. Zhu, and C. A. Mirkin, Observation of surfaceinduced broken time-reversal symmetry in $\mathrm{yba}_{2} \mathrm{Cu}_{3} \mathrm{O}_{7}$ tunnel junctions, Phys. Rev. Lett. 79, 277 (1997)

[8] L. Alff, H. Takashima, S. Kashiwaya, N. Terada, H. Ihara, Y. Tanaka, M. Koyanagi, and K. Kajimura, Spatially continuous zero-bias conductance peak on (110) $\mathrm{YBa}_{2} \mathrm{Cu}_{3} \mathrm{O}_{7-\delta}$ surfaces, Phys. Rev. B 55, R14757 (1997).

[9] J. Y. T. Wei, N.-C. Yeh, D. F. Garrigus, and M. Strasik, Directional tunneling and andreev reflection on $\mathrm{YBa}_{2} \mathrm{Cu}_{3} \mathrm{O}_{7-\delta}$ single crystals: Predominance of $d$-wave pairing symmetry verified with the generalized blonder, tinkham, and klapwijk theory, Phys. Rev. Lett. 81, 2542 (1998)

[10] Y. Tanaka, M. Sato, and N. Nagaosa, Symmetry and topology in superconductors odd-frequency pairing and edge states-, Journal of the Physical Society of Japan 81, 011013 (2012) https://doi.org/10.1143/JPSJ.81.011013.

[11] S. Ryu and Y. Hatsugai, Topological origin of zeroenergy edge states in particle-hole symmetric systems, Phys. Rev. Lett. 89, 077002 (2002)

[12] A. P. Schnyder, S. Ryu, A. Furusaki, and A. W. W. Ludwig, Classification of topological insulators and superconductors in three spatial dimensions, Phys. Rev. B 78, 195125 (2008)

[13] M. Sato, Y. Tanaka, K. Yada, and T. Yokoyama, Topology of andreev bound states with flat dispersion, Phys. Rev. B 83, 224511 (2011)

[14] M. Sato and S. Fujimoto, Majorana fermions and topology in superconductors, Journal of the Physical Society of Japan 85, 072001 (2016) https://doi.org/10.7566/JPSJ.85.072001.

[15] M. Sato and Y. Ando, Topological superconductors: a review, Reports on Progress in Physics 80, 076501 (2017).

[16] M. Sato, Nodal structure of superconductors with time-reversal invariance and $\boldsymbol{Z}_{2}$ topological number, Phys. Rev. B 73, 214502 (2006)

[17] S. Kobayashi, K. Shiozaki, Y. Tanaka, and M. Sato, Topological blount's theorem of odd-parity superconductors, Phys. Rev. B 90, 024516 (2014)

[18] V. L. Berezinskii, New model of the anisotropic phase of superfluid $\mathrm{He}^{3}$, JETP Lett. 20, 287 (1974).

[19] J. Linder and A. V. Balatsky, arXiv:1709.03986.

[20] Y. Tanaka, A. A. Golubov, S. Kashiwaya, and M. Ueda, Anomalous josephson effect between even- and odd-frequency superconductors,
Phys. Rev. Lett. 99, 037005 (2007)

[21] M. Eschrig, T. Löfwander, T. Champel, J. C. Cuevas, J. Kopu, and G. Schön, Symmetries of Pairing Correlations in Superconductor-Ferromagnet Nanostructures, Journal of Low Temperature Physics 147, 457 (2007).

[22] Y. Tanaka, Y. Tanuma, and A. A. Golubov, Oddfrequency pairing in normal-metal/superconductor junctions, Phys. Rev. B 76, 054522 (2007)

[23] C. Triola, D. M. Badiane, A. V. Balatsky, and E. Rossi, General conditions for proximity-induced odd-frequency superconductivity in two-dimensional electronic systems, Phys. Rev. Lett. 116, 257001 (2016)

[24] D. Kuzmanovski and A. M. Black-Schaffer, Multiple odd-frequency superconducting states in buckled quantum spin hall insulators with time-reversal symmetry, Phys. Rev. B 96, 174509 (2017)

[25] Y. Tanaka and A. A. Golubov, Theory of the proximity effect in junctions with unconventional superconductors, Phys. Rev. Lett. 98, 037003 (2007).

[26] Y. Tanaka and S. Kashiwaya, Anomalous charge transport in triplet superconductor junctions, Phys. Rev. B 70, 012507 (2004)

[27] Y. Tanaka, S. Kashiwaya, and T. Yokoyama, Theory of enhanced proximity effect by midgap andreev resonant state in diffusive normal-metal/triplet superconductor junctions, Phys. Rev. B 71, 094513 (2005)

[28] Y. Asano, Y. Tanaka, and S. Kashiwaya, Anomalous josephson effect in $p$-wave dirty junctions, Phys. Rev. Lett. 96, 097007 (2006)

[29] Y. Asano, Y. Tanaka, A. A. Golubov, and S. Kashiwaya, Phys. Rev. Lett. 99, 067005 (2007).

[30] A. A. Golubov and M. Y. Kupriyanov, Theoretical investigation of josephson tunnel junctions with spatially inhomogeneous superconducting electrodes, Journal of Low Temperature Physics 70, 83 (1988).

[31] S. Ikegaya, S.-I. Suzuki, Y. Tanaka, and Y. Asano, Quantization of conductance minimum and index theorem, Phys. Rev. B 94, 054512 (2016)

[32] G. Annunziata, D. Manske, and J. Linder, Proximity effect with noncentrosymmetric superconductors, Phys. Rev. B 86, 174514 (2012)

[33] S. Higashitani, Y. Nagato, and K. Nagai, Proximity Effect between a Dirty Fermi Liquid and Superfluid ${ }^{3} \mathrm{He}$, Journal of Low Temperature Physics 155, 83 (2009).

[34] S. Higashitani, S. Matsuo, Y. Nagato, K. Nagai, S. Murakawa, R. Nomura, and Y. Okuda, Odd-frequency cooper pairs and zero-energy surface bound states in superfluid ${ }^{3}$ He, Phys. Rev. B 85, 024524 (2012)

[35] S. V. Bakurskiy, A. A. Golubov, M. Y. Kupriyanov, K. Yada, and Y. Tanaka, Anomalous surface states at interfaces in $p$-wave superconductors, Phys. Rev. B 90, 064513 (2014)

[36] S. Higashitani, Mechanism of paramagnetic meissner effect in high-temperature superconductors, Journal of the Physical Society of Japan 66, 2556 (1997)

[37] H. Walter, W. Prusseit, R. Semerad, H. Kinder, W. Assmann, H. Huber, H. Burkhardt, D. Rainer, and J. A. Sauls, Low-temperature anomaly in the penetration depth of $\mathrm{YBa}_{2} \mathrm{Cu}_{3} \mathrm{O}_{7}$ films: Evidence for andreev bound states at surfaces, Phys. Rev. Lett. 80, 3598 (1998)

[38] Y. Tanaka, Y. Asano, A. A. Golubov, and S. Kashiwaya, Anomalous features of the proximity effect in triplet superconductors, Phys. Rev. B 72, 140503(R) (2005).

[39] T. Yokoyama, Y. Tanaka, and N. Nagaosa, 
Anomalous meissner effect in a normalmetal ${ }^{`}$ superconductor junction with a spin-active interface, Phys. Rev. Lett. 106, 246601 (2011).

[40] S.-I. Suzuki and Y. Asano, Paramagnetic instability of small topological superconductors, Phys. Rev. B 89, 184508 (2014)

[41] S.-I. Suzuki and Y. Asano, Effects of surface roughness on the paramagnetic response of small unconventional superconductors, Phys. Rev. B 91, 214510 (2015).

[42] S.-I. Suzuki and Y. Asano, Spontaneous edge current in a small chiral superconductor with a rough surface, Phys. Rev. B 94, 155302 (2016)

[43] S.-P. Lee, R. M. Lutchyn, and J. Maciejko, Oddfrequency superconductivity in a nanowire coupled to majorana zero modes, Phys. Rev. B 95, 184506 (2017)

[44] Y. Asano, A. A. Golubov, Y. V. Fominov, and Y. Tanaka, Unconventional surface impedance of a normal-metal film covering a spin-triplet superconductor due to odd-frequency cooper pairs, Phys. Rev. Lett. 107, 087001 (2011)

[45] S. V. Bakurskiy, Y. V. Fominov, A. F. Shevchun, Y. Asano, Y. Tanaka, M. Y. Kupriyanov, A. A. Golubov, M. R. Trunin, H. Kashiwaya, S. Kashiwaya, and Y. Maeno, Local impedance on a rough surface of a chiral $p$-wave superconductor, Phys. Rev. B 98, 134508 (2018)

[46] Y. Asano and Y. Tanaka, Majorana fermions and oddfrequency cooper pairs in a normal-metal nanowire proximity-coupled to a topological superconductor, Phys. Rev. B 87, 104513 (2013).

[47] S. Ikegaya, Y. Asano, and Y. Tanaka, Anomalous proximity effect and theoretical design for its realization, Phys. Rev. B 91, 174511 (2015).

[48] S. Ikegaya, S. Kobayashi, and Y. Asano, Symmetry conditions of a nodal superconductor for generating robust flat-band andreev bound states at its dirty surface, Phys. Rev. B 97, 174501 (2018)

[49] V. Stanev and V. Galitski, Quasiclassical eilenberger theory of the topological proximity effect in a superconducting nanowire, Phys. Rev. B 89, 174521 (2014).

[50] H. Ebisu, K. Yada, H. Kasai, and Y. Tanaka, Odd-frequency pairing in topological superconductivity in a one-dimensional magnetic chain, Phys. Rev. B 91, 054518 (2015).

[51] X. Liu, J. D. Sau, and S. Das Sarma, Universal spin-triplet superconducting correlations of majorana fermions, Phys. Rev. B 92, 014513 (2015).

[52] H. Ebisu, B. Lu, K. Taguchi, A. A. Golubov, and Y. Tanaka, Josephson current in a normal-metal nanowire coupled to a superconductor/ferromagnet/superconductor junction, Phys. Rev. B 93, 024509 (2016)

[53] H. Ebisu, B. Lu, J. Klinovaja, and Y. Tanaka, Theory of time-reversal topological superconductivity in double rashba wires: symmetries of cooper pairs and andreev bound states, Progress of Theoretical and Experimental Physics 2016,

[54] J. Cayao and A. M. Black-Schaffer, Odd-frequency superconducting pairing and subgap density of states at the edge of a two-dimensional topological insulator without magnetism, Phys. Rev. B 96, 155426 (2017).

[55] A. M. Black-Schaffer and A. V. Balatsky, Proximityinduced unconventional superconductivity in topological insulators, Phys. Rev. B 87, 220506(R) (2013)
[56] F. Keidel, P. Burset, and B. Trauzettel, Tunable hybridization of majorana bound states at the quantum spin hall edge, Phys. Rev. B 97, 075408 (2018)

[57] D. Breunig, P. Burset, and B. Trauzettel, Creation of spin-triplet cooper pairs in the absence of magnetic ordering, Phys. Rev. Lett. 120, 037701 (2018)

[58] F. Crépin, P. Burset, and B. Trauzettel, Odd-frequency triplet superconductivity at the helical edge of a topological insulator, Phys. Rev. B 92, 100507(R) (2015).

[59] O. Kashuba, B. Sothmann, P. Burset, and B. Trauzettel, Majorana STM as a perfect detector of odd-frequency superconductivity, Phys. Rev. B 95, 174516 (2017).

[60] F. S. Bergeret, A. F. Volkov, and K. B. Efetov, Longrange proximity effects in superconductor-ferromagnet structures, Phys. Rev. Lett. 86, 4096 (2001)

[61] F. S. Bergeret, A. F. Volkov, and K. B. Efetov, Odd triplet superconductivity and related phenomena in superconductor-ferromagnet structures, Rev. Mod. Phys. 77, 1321 (2005)

[62] A. F. Volkov, F. S. Bergeret, and K. B. Efetov, Odd triplet superconductivity in superconductor-ferromagnet multilayered structures, Phys. Rev. Lett. 90, 117006 (2003).

[63] M. Eschrig, Spin-polarized supercurrents for spintronics, Phys. Today 64, 43 (2011).

[64] M. Eschrig, J. Kopu, J. C. Cuevas, and G. Schön, Theory of half-metal/superconductor heterostructures, Phys. Rev. Lett. 90, 137003 (2003).

[65] Y. Asano, Y. Tanaka, and A. A. Golubov, Josephson effect due to odd-frequency pairs in diffusive half metals, Phys. Rev. Lett. 98, 107002 (2007)

[66] V. Braude and Y. V. Nazarov, Fully developed triplet proximity effect, Phys. Rev. Lett. 98, 077003 (2007).

[67] T. Yokoyama, Y. Tanaka, and A. A. Golubov, Manifestation of the odd-frequency spin-triplet pairing state in diffusive ferromagnet/superconductor junctions, Phys. Rev. B 75, 134510 (2007)

[68] Y. Asano, Y. Sawa, Y. Tanaka, and A. A. Golubov, Odd-frequency pairs and josephson current through a strong ferromagnet, Phys. Rev. B 76, 224525 (2007)

[69] M. Eschrig and T. Löfwander, Triplet supercurrents in clean and disordered half-metallic ferromagnets, Nature Physics 4, 138 (2008).

[70] M. Eschrig, Spin-polarized supercurrents for spintronics: a review of current progress, Reports on Progress in Physics 78, 104501 (2015)

[71] M. Alidoust, K. Halterman, and O. T. Valls, Zeroenergy peak and triplet correlations in nanoscale superconductor/ferromagnet/ferromagnet spin valves, Phys. Rev. B 92, 014508 (2015)

[72] E. Bauer, G. Hilscher, H. Michor, C. Paul, E. W. Scheidt, A. Gribanov, Y. Seropegin, H. Noël, M. Sigrist, and P. Rogl, Heavy Fermion Superconductivity and Magnetic Order in Noncentrosymmetric $\mathrm{CePt}_{3} \mathrm{Si}$, Phys. Rev. Lett. 92, 027003 (2004).

[73] N. Reyren, S. Thiel, A. D. Caviglia, L. F. Kourkoutis, 1 (Q0116ammerl, C. Richter, C. W. Schneider, T. Kopp, A.S. Rüetschi, D. Jaccard, M. Gabay, D. A. Muller, J.-M. Triscone, and J. Mannhart, Superconducting interfaces between insulating oxides, Science 317, 1196 (2007)

[74] L. P. Gor'kov and E. I. Rashba, Superconducting 2D System with Lifted Spin Degeneracy: Mixed SingletTriplet State, Phys. Rev. Lett. 87, 037004 (2001).

[75] P. A. Frigeri, D. F. Agterberg, A. Koga, and M. Sigrist, 
Superconductivity without inversion symmetry: $\mathrm{MnSi}$ versus $\mathrm{CePt}_{3} \mathrm{Si}$, Phys. Rev. Lett. 92, 097001 (2004)

[76] S. Fujimoto, Electron Correlation and Pairing States in Superconductors without Inversion Symmetry, Journal of the Physical Society of Japan 76, 051008 (2007)

[77] M. Sato and S. Fujimoto, Topological phases of noncentrosymmetric superconductors: Edge states, majorana fermions, and non-abelian statistics, Phys. Rev. B 79, 094504 (2009)

[78] C. R. Reeg and D. L. Maslov, Proximity-induced triplet superconductivity in rashba materials, Phys. Rev. B 92, 134512 (2015)

[79] I. V. Bobkova and A. M. Bobkov, Quasiclassical theory of magnetoelectric effects in superconducting heterostructures in the presence of spin-orbit coupling, Phys. Rev. B 95, 184518 (2017).

[80] J. Cayao and A. M. Black-Schaffer, Odd-frequency superconducting pairing in junctions with rashba spinorbit coupling, Phys. Rev. B 98, 075425 (2018)

[81] S. Kobayashi, Y. Tanaka, and M. Sato, Fragile surface zero-energy flat bands in three-dimensional chiral superconductors, Phys. Rev. B 92, 214514 (2015)

[82] J. C. Budich and E. Ardonne, Topological invariant for generic one-dimensional timereversal-symmetric superconductors in class diii, Phys. Rev. B 88, 134523 (2013)

[83] Y. Tanaka, Y. V. Nazarov, and S. Kashiwaya, Circuit theory of unconventional superconductor junctions, Phys. Rev. Lett. 90, 167003 (2003)

[84] Y. Tanaka, Y. V. Nazarov, A. A. Golubov, and S. Kashiwaya, Theory of charge transport in diffusive normal metal/unconventional singlet superconductor contacts, Phys. Rev. B 69, 144519 (2004)

[85] P. M. C. Rourke, M. A. Tanatar, C. S. Turel, J. Berdeklis, C. Petrovic, and J. Y. T. Wei, Spectroscopic evidence for multiple order parameter components in the heavy fermion superconductor $\mathrm{CeCoIn}_{5}$, Phys. Rev. Lett. 94, 107005 (2005)

[86] M. Shimozawa, S. K. Goh, R. Endo, R. Kobayashi, T. Watashige, Y. Mizukami, H. Ikeda, H. Shishido, Y. Yanase, T. Terashima, T. Shibauchi, and Y. Matsuda, Controllable Rashba Spin-Orbit Interaction in Artificially Engineered Superlattices Involving the Heavy-Fermion Superconductor CeCoIn 5 , Phys. Rev. Lett. 112, 156404 (2014)

[87] Y. Nagato and K. Nagai, Surface and size effect of a $d_{x y^{-}}$ state superconductor, Phys. Rev. B 51, 16254 (1995).

[88] L. J. Buchholtz, M. Palumbo, D. Rainer, and J. A. Sauls, Thermodynamics of a $d$-wave Superconductor Near a Surface, Journal of Low Temperature Physics 101, 1079 (1995)

[89] Y. S. Barash, A. A. Svidzinsky, and H. Burkhardt, Quasiparticle bound states and low-temperature peaks of the conductance of nis junctionsin d-wave superconductors, Phys. Rev. B 55, 15282 (1997)

[90] Y. Tanuma, Y. Tanaka, and S. Kashiwaya, Tunneling conductance of normal metal $/ d_{x^{2}-y^{2}}$-wave superconductor junctions in the presence of broken time-reversal symmetry states near interfaces, Phys. Rev. B 64, 214519 (2001)

[91] K. Yamada, Y. Nagato, S. Higashitani, and K. Nagai, Rough Surface Effects on $d$-Wave Superconductors, Journal of the Physical Society of Japan 65, 1540 (1996)

[92] A. A. Golubov and M. Y. Kupriyanov, Surface electron scattering in d-wave superconductors, Journal of Experimental and Theoretical Physics Letters 69, 262

[93] B. Lu, P. Burset, Y. Tanuma, A. A. Golubov, Y. Asano, and Y. Tanaka, Influence of the impurity scattering on charge transport in unconventional superconductor junctions, Phys. Rev. B 94, 014504 (2016)

[94] S. Wu and K. V. Samokhin, Tunneling into $d$-wave superconductors: Effects of interface spin-orbit coupling, Phys. Rev. B 82, 184501 (2010).

[95] S. Wu and K. V. Samokhin, Effects of interface spin-orbit coupling on tunneling between normal metal and chiral $p$-wave superconductor, Phys. Rev. B 81, 214506 (2010).

[96] S. H. Jacobsen, J. A. Ouassou, and J. Linder, Critical temperature and tunneling spectroscopy of superconductor-ferromagnet hybrids with intrinsic rashba-dresselhaus spin-orbit coupling, Phys. Rev. B 92, 024510 (2015).

[97] G. C. Paul, P. Dutta, and A. Saha, Transport and noise properties of a normal metalsuperconductor-normal metal junction with mixed singlet and chiral triplet pairings, Journal of Physics: Condensed Matter 29, 015301 (2017)

[98] T. R. Kirkpatrick and D. Belitz, Disorder-induced triplet superconductivity, Phys. Rev. Lett. 66, 1533 (1991)

[99] D. Belitz and T. R. Kirkpatrick, Even-parity spintriplet superconductivity in disordered electronic systems, Phys. Rev. B 46, 8393 (1992).

[100] A. Balatsky and E. Abrahams, New class of singlet superconductors which break the time reversal and parity, Phys. Rev. B 45, 13125 (1992).

[101] V. J. Emery and S. Kivelson, Mapping of the twochannel Kondo problem to a resonant-level model, Phys. Rev. B 46, 10812 (1992)

[102] P. Coleman, E. Miranda, and A. Tsvelik, Odd-frequency pairing in the Kondo lattice, Phys. Rev. B 49, 8955 (1994)

[103] E. Abrahams, A. Balatsky, D. J. Scalapino, and J. R. Schrieffer, Properties of odd-gap superconductors, Phys. Rev. B 52, 1271 (1995)

[104] P. Coleman, A. Georges, and A. M. Tsvelik, Reflections on the one-dimensional realization of odd-frequency pairing, Journal of Physics: Condensed Matter 9, 345 (1997).

[105] D. Belitz and T. R. Kirkpatrick, Properties of spin-triplet, even-parity superconductors, Phys. Rev. B 60, 3485 (1999)

[106] M. Vojta and E. Dagotto, Indications of unconventional superconductivity in doped and undoped triangular antiferromagnets, Phys. Rev. B 59, R713 (1999).

[107] Y. Fuseya, H. Kohno, and K. Miyake, Realization of odd-frequency p-wave spin-singlet superconductivity coexisting with antiferromagnetic order near quantum critical point, Journal of the Physical Society of Japan 72, 2914 (2003)

[108] K. Shigeta, S. Onari, K. Yada, and Y. Tanaka, Theory of odd-frequency pairings on a quasione-dimensional lattice in the hubbard model, Phys. Rev. B 79, 174507 (2009).

[109] T. Hotta, Double-exchange ferromagnetism and orbital-fluctuation-induced superconductivity in cubic uranium compounds, Journal of the Physical Society of Japan 78, 123710 (2009) 
[110] K. Shigeta, Y. Tanaka, K. Kuroki, S. Onari, and H. Aizawa, Competition of pairing symmetries and a mechanism for berezinskii pairing in quasi-one-dimensional systems, Phys. Rev. B 83, 140509(R) (2011).

[111] D. Solenov, I. Martin, and D. Mozyrsky, Thermodynamical stability of odd-frequency superconducting state, Phys. Rev. B 79, 132502 (2009)

[112] Y. Fuseya and K. Miyake, On the meissner effect of the odd-frequency superconductivity with critical spin fluctuations: Possibility of zero field fflo pairing, Journal of the Physical Society of Japan 80, 054705 (2011)

[113] H. Kusunose, Y. Fuseya, and K. Miyake, On the puzzle of odd-frequency superconductivity, Journal of the Physical Society of Japan 80, 054702 (2011)

[114] H. Kusunose, Y. Fuseya, and K. Miyake, Possible odd-frequency superconductivity in strong-coupling electron-phonon systems, Journal of the Physical Society of Japan 80, 044711 (2011)

[115] K. Fukui and Y. Kato, Mixing effect of even- frequency on odd-frequency pairings in strongly correlated electron systems under magnetic field, Journal of the Physical Society of Japan 87, 014706 (2018)

[116] Y. Asano, Y. V. Fominov, and Y. Tanaka, Consequences of bulk odd-frequency superconducting states for the classification of cooper pairs, Phys. Rev. B 90, 094512 (2014)

[117] Y. V. Fominov, Y. Tanaka, Y. Asano, and M. Eschrig, Odd-frequency superconducting states with different types of meissner response: Problem of coexistence, Phys. Rev. B 91, 144514 (2015).

[118] S. Hoshino, Mean-field description of odd-frequency superconductivity with staggered ordering vector, Phys. Rev. B 90, 115154 (2014)

[119] S. Hoshino, K. Yada, and Y. Tanaka, Tunneling and Josephson effects in odd-frequency superconductor junctions: A study on multichannel Kondo chain, Phys. Rev. B 93, 224511 (2016).

[120] A. Umerski, Closed-form solutions to surface Green's functions, Phys. Rev. B 55, 5266 (1997)

\section{Appendix A: Derivation of surface Green's function}

In this Appendix, we explain a recursive method to calculate the Green's function [120]. The Hamiltonian with open boundary condition in the $x$-direction and periodic boundary condition in the $y$-direction is given by

$$
\begin{aligned}
& \mathcal{H}_{n, \mathrm{SC}}=\sum_{k_{y}} C_{n, k_{y}}^{\dagger} H_{n}\left(k_{y}\right) C_{n, k_{y}}, \\
& H_{n}\left(k_{y}\right)=\left(\begin{array}{ccccc}
\hat{u} & \hat{t} & & & \\
\hat{t}^{\dagger} & \hat{u} & \hat{t} & & \\
& \hat{t}^{\dagger} & \ddots & \ddots & \\
& & & \ddots & \hat{t} \\
& & & \hat{t}^{\dagger} & \hat{u}
\end{array}\right), \\
& \hat{u}=\left(\begin{array}{cccc}
\varepsilon_{k_{y}} & \lambda \sin k_{y} & & \\
\lambda \sin k_{y} & \varepsilon_{k_{y}} & & \\
& & -\varepsilon_{k_{y}} & \lambda \sin k_{y} \\
& & \lambda \sin k_{y} & -\varepsilon_{k_{y}}
\end{array}\right), \\
& \hat{t}=\left(\begin{array}{cccc}
-t & \lambda / 2 & 0 & \Delta_{12}\left(k_{y}\right) \\
-\lambda / 2 & -t & \Delta_{21}\left(k_{y}\right) & 0 \\
0 & \Delta_{21}\left(k_{y}\right) & t & -\lambda / 2 \\
\Delta_{12}\left(k_{y}\right) & 0 & \lambda / 2 & t
\end{array}\right),
\end{aligned}
$$

where $n$ is the number of sites in the $x$-direction, $\varepsilon_{k_{y}}=-2 t \cos k_{y}-\mu, \Delta_{12}$ and $\Delta_{21}$ are given by

$$
\begin{aligned}
& \Delta_{12}\left(k_{y}\right)=\Delta_{0} /(2 i), \\
& \Delta_{21}\left(k_{y}\right)=\Delta_{0} /(2 i),
\end{aligned}
$$

for the spin-triplet $p_{x}$-wave case, $\Delta_{12}$ and $\Delta_{21}$ are given by

$$
\begin{aligned}
& \Delta_{12}\left(k_{y}\right)=-i \Delta_{0} \sin k_{y} / 2, \\
& \Delta_{21}\left(k_{y}\right)=i \Delta_{0} \sin k_{y} / 2,
\end{aligned}
$$

for the spin-singlet $d_{x y}$-wave case and

$$
C_{n, k_{y}}=\left(c_{1, k_{y}, \uparrow}, c_{1, k_{y}, \downarrow}, c_{1,-k_{y}, \uparrow}^{\dagger}, c_{1,-k_{y}, \downarrow}^{\dagger}, \ldots, c_{n, k_{y}, \uparrow}, c_{n, k_{y}, \downarrow}, c_{n,-k_{y}, \uparrow}^{\dagger} c_{n,-k_{y}, \downarrow}^{\dagger}\right)^{\mathrm{T}} .
$$


Let Möbius transformation be

$$
\hat{A}_{\bullet} \hat{z}=(\hat{a} \hat{z}+\hat{b})(\hat{c} \hat{z}+\hat{d})^{-1}
$$

with

$$
\hat{A}=\left(\begin{array}{ll}
\hat{a} & \hat{b} \\
\hat{c} & \hat{d}
\end{array}\right)
$$

where $\hat{a}, \hat{b}, \hat{c}, \hat{d}$ and $\hat{z}$ are $m \times m$ matrices. The Möbius transformation satisfies following relation:

$$
\hat{A}_{\bullet} \hat{B} \bullet \hat{z}=(\hat{A} \hat{B}) . \hat{z}
$$

We also define the elements of the Green's function as

$$
\left[z-H_{n}\left(k_{y}\right)\right]^{-1}=\left(\begin{array}{ccc}
{\left[g_{n}\left(z, k_{y}\right)\right]_{1,1}} & {\left[g_{n}\left(z, k_{y}\right)\right]_{1,2}} & \cdots \\
{\left[g_{n}\left(z, k_{y}\right)\right]_{2,1}} & {\left[g_{n}\left(z, k_{y}\right)\right]_{2,2}} & \\
\vdots & & \ddots
\end{array}\right),
$$

with $4 \times 4$ matrix $\left[g_{n}\left(z, k_{y}\right)\right]_{j_{x}, j_{x}^{\prime}}$. Then the recurrence relation for the surface Green's function for the $n$-site system at the leftmost site $\left[g_{n}\left(z, k_{y}\right)\right]_{1,1}$ and the $n+1$-site one $\left[g_{n+1}\left(z, k_{y}\right)\right]_{1,1}$ is given by

$$
\begin{aligned}
{\left[g_{n+1}\left(z, k_{y}\right)\right]_{1,1} } & =\left\{(z-\hat{u})-\hat{t}\left[g_{n}\left(z, k_{y}\right)\right]_{1,1} \hat{t}^{\dagger}\right\}^{-1} \\
& =X \bullet\left[g_{n}\left(z, k_{y}\right)\right]_{1,1},
\end{aligned}
$$

with

$$
X=\left(\begin{array}{cc}
\hat{0} & \left(\hat{t}^{\dagger}\right)^{-1} \\
-\hat{t} & (z-\hat{u})\left(\hat{t}^{\dagger}\right)^{-1}
\end{array}\right) .
$$

Here, $\hat{0}$ is a $4 \times 4$ matrix with all the elements equal to zero. It is noted $\left[g_{1}\left(z, k_{y}\right)\right]_{1,1}$ satisfies

$$
\left[g_{1}\left(z, k_{y}\right)\right]_{1,1}=X \bullet \hat{0}
$$

and $\left[g_{n}\left(z, k_{y}\right)\right]_{1,1}$ is written as

$$
\left[g_{n}\left(z, k_{y}\right)\right]_{1,1}=\left(X^{n}\right) . \hat{0} .
$$

Let $U$ be a matrix which diagonalizes $X$ as

$$
U^{-1} X U=\operatorname{diag}\left(\lambda_{1}, \lambda_{2}, \ldots, \lambda_{8}\right),
$$

with $\left|\lambda_{1}\right| \leq \cdots \leq\left|\lambda_{4}\right|<\left|\lambda_{5}\right| \leq \cdots \leq\left|\lambda_{8}\right|$. Then the surface Green's function for a semi-infinite system at the leftmost site $G_{j_{x}=1}^{\infty}\left(z, k_{y}\right)$ is given by

$$
\begin{aligned}
G_{j_{x}=1}^{\infty}\left(z, k_{y}\right) & =\lim _{n \rightarrow \infty}\left[g_{n}\left(z, k_{y}\right)\right]_{1,1} \\
& =\lim _{n \rightarrow \infty}\left[U\left(U^{-1} X U\right)^{n} U^{-1}\right] \bullet^{\hat{0}} \\
& =U \bullet \hat{0} \\
& =U_{12}\left(U_{22}\right)^{-1},
\end{aligned}
$$

where $U_{i j}$ is a $4 \times 4$ matrix defined as

$$
U=\left(\begin{array}{ll}
U_{11} & U_{12} \\
U_{21} & U_{22}
\end{array}\right),
$$

and we use Eq. (A10). 


\section{Appendix B: Winding number for spin-singlet $d_{x y}$-wave superconductor with RSOC}

From Eq. (9), point nodes are on $k_{x(y)}=0$ or $k_{x(y)}=\pi$, since $\Delta_{0} \sin k_{x} \sin k_{y}=0$ is satisfied at these points. We consider only $k_{x}=0$ or $k_{x}=\pi$ case because Eq. (9) is symmetric with respect to replacing $k_{x}$ by $k_{y}$. Point nodes also satisfy $\xi_{k} \pm\left|\lambda\left(\sin k_{x}+\sin k_{y}\right)\right|=0$. For $k_{x}=0$, point nodes are located at

$$
k_{y}=k_{0, \pm}=\arccos \left\{\frac{1}{4 t^{2}+\lambda^{2}}\left[-2 t(2 t+\mu) \pm \lambda \sqrt{\lambda^{2}-4 t \mu-\mu^{2}}\right]\right\}
$$

with $\lambda^{2}-4 t \mu-\mu^{2} \geq 0$ and for $k_{x}=\pi$, point nodes are located at

$$
k_{y}=k_{\pi, \pm}=\arccos \left\{\frac{1}{4 t^{2}+\lambda^{2}}\left[-2 t(-2 t+\mu) \pm \lambda \sqrt{\lambda^{2}+4 t \mu-\mu^{2}}\right]\right\}
$$

with $\lambda^{2}+4 t \mu-\mu^{2} \geq 0$. Here we suppose $\lambda \geq 0$. Then the number of point nodes is

$$
\begin{cases}8 & -2 t-\sqrt{4 t^{2}+\lambda^{2}}<\mu<+2 t-\sqrt{4 t^{2}+\lambda^{2}} \\ 16 & +2 t-\sqrt{4 t^{2}+\lambda^{2}}<\mu<-2 t+\sqrt{4 t^{2}+\lambda^{2}} \\ 8 & -2 t+\sqrt{4 t^{2}+\lambda^{2}}<\mu<+2 t+\sqrt{4 t^{2}+\lambda^{2}}\end{cases}
$$

with the non-zero value of the RSOC.

As shown in Fig. 14, there are nine regimes from A to I. The region A in Fig. 14, the winding number is zero for

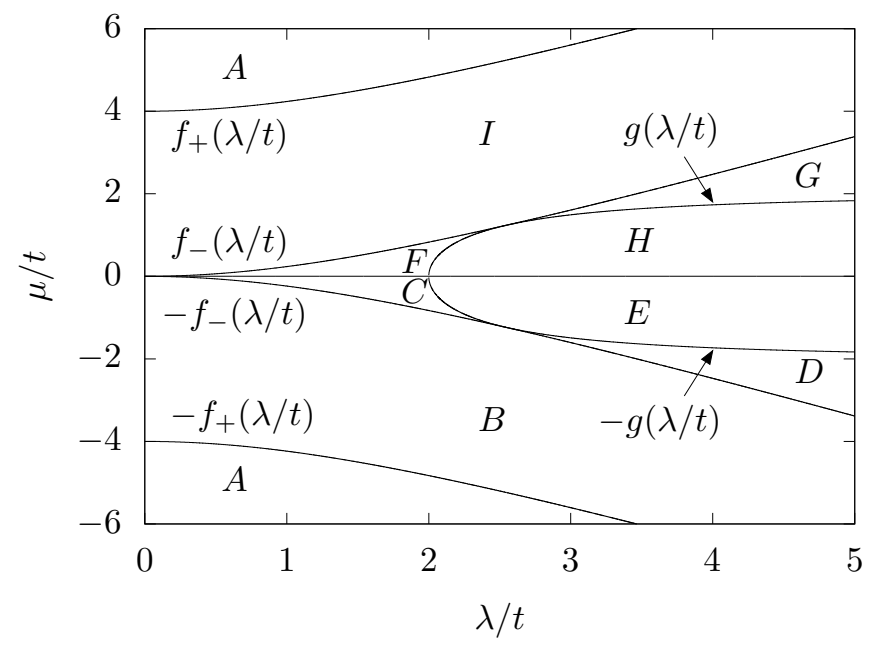

FIG. 14. Zero-energy flatband is characterized by the region A to I. $f_{ \pm}(\lambda / t)= \pm 2+\sqrt{4^{2}+(\lambda / t)^{2}}$, and $g(\lambda / t)=$ $\frac{2}{\lambda / t} \sqrt{(\lambda / t)^{2}-4^{2}}$

any $k_{y}$.

The winding number for $-2 t-\sqrt{4 t^{2}+\lambda^{2}}<\mu<+2 t-\sqrt{4 t^{2}+\lambda^{2}}$ [region B in Fig. 14] is

$$
W\left(k_{y}\right)= \begin{cases}0 & -\pi<k_{y}<-k_{0,-} \\ -1 & -k_{0,-}<k_{y}<-k_{0,+} \\ -2 & -k_{0,+}<k_{y}<0 \\ 2 & 0<k_{y}<k_{0,+} \\ 1 & k_{0,+}<k_{y}<k_{0,-} \\ 0 & k_{0,-}<k_{y}<\pi\end{cases}
$$


The winding number for $+2 t-\sqrt{4 t^{2}+\lambda^{2}}<\mu<0$ and $\lambda<\sqrt{2(1+\sqrt{5})} t$ [region C in Fig. 14] is

$$
W\left(k_{y}\right)= \begin{cases}0 & -\pi<k_{y}<-k_{0,-}, \\ -1 & -k_{0,-}<k_{y}<-k_{0,+}, \\ -2 & -k_{0,+}<k_{y}<-k_{\pi,-}, \\ -1 & -k_{\pi,-}<k_{y}<-k_{\pi,+}, \\ -2 & -k_{\pi,+}<k_{y}<0 \\ 2 & 0<k_{y}<k_{\pi,+} \\ 1 & k_{\pi,+}<k_{y}<k_{\pi,-}, \\ 2 & k_{\pi,-}<k_{y}<k_{0,+}, \\ 1 & k_{0,+}<k_{y}<k_{0,-} \\ 0 & k_{0,-}<k_{y}<\pi\end{cases}
$$

The winding number for $+2 t-\sqrt{4 t^{2}+\lambda^{2}}<\mu<-\frac{2 t}{\lambda} \sqrt{\lambda^{2}-4 t^{2}}$ and $\lambda>\sqrt{2(1+\sqrt{5})} t$ [region D in Fig. 14] is

$$
W\left(k_{y}\right)= \begin{cases}0 & -\pi<k_{y}<-k_{0,-}, \\ -1 & -k_{0,-}<k_{y}<-k_{\pi,-}, \\ 0 & -k_{\pi,-}<k_{y}<-k_{\pi,+}, \\ -1 & -k_{\pi,+}<k_{y}<-k_{0,+}, \\ -2 & -k_{0,+}<k_{y}<0 \\ 2 & 0<k_{y}<k_{0,+} \\ 1 & k_{0,+}<k_{y}<k_{\pi,+}, \\ 0 & k_{\pi,+}<k_{y}<k_{\pi,-}, \\ 1 & k_{\pi,-}<k_{y}<k_{0,-}, \\ 0 & k_{0,-}<k_{y}<\pi\end{cases}
$$

The winding number for $-\frac{2 t}{\lambda} \sqrt{\lambda^{2}-4 t^{2}}<\mu<0$ [region E in Fig. 14] is

$$
W\left(k_{y}\right)= \begin{cases}0 & -\pi<k_{y}<-k_{0,-}, \\ -1 & -k_{0,-}<k_{y}<-k_{\pi,-}, \\ 0 & -k_{\pi,-}<k_{y}<-k_{0,+}, \\ -1 & -k_{0,+}<k_{y}<-k_{\pi,+}, \\ -2 & -k_{\pi,+}<k_{y}<0 \\ 2 & 0<k_{y}<k_{\pi,+} \\ 1 & k_{\pi,+}<k_{y}<k_{0,+} \\ 0 & k_{0,+}<k_{y}<k_{\pi,-}, \\ 1 & k_{\pi,-}<k_{y}<k_{0,-}, \\ 0 & k_{0,-}<k_{y}<\pi\end{cases}
$$

The winding number for $0<\mu<-2 t+\sqrt{4 t^{2}+\lambda^{2}}$ and $\lambda<\sqrt{2(1+\sqrt{5})} t$ [region F in Fig. [14] is

$$
W\left(k_{y}\right)= \begin{cases}-2 & -\pi<k_{y}<-k_{0,-}, \\ -1 & -k_{0,-}<k_{y}<-k_{0,+}, \\ -2 & -k_{0,+}<k_{y}<-k_{\pi,-}, \\ -1 & -k_{\pi,-}<k_{y}<-k_{\pi,+}, \\ 0 & -k_{\pi,+}<k_{y}<k_{\pi,+}, \\ 1 & k_{\pi,+}<k_{y}<k_{\pi,-}, \\ 2 & k_{\pi,-}<k_{y}<k_{0,+}, \\ 1 & k_{0,+}<k_{y}<k_{0,-}, \\ 2 & k_{0,-}<k_{y}<\pi .\end{cases}
$$


The winding number for $0<\mu<-2 t+\sqrt{4 t^{2}+\lambda^{2}}$ and $\lambda>\sqrt{2(1+\sqrt{5})} t$ [region G in Fig. 14] is

$$
W\left(k_{y}\right)= \begin{cases}-2 & -\pi<k_{y}<-k_{\pi,-}, \\ -1 & -k_{\pi,-}<k_{y}<-k_{0,-}, \\ 0 & -k_{0,-}<k_{y}<-k_{0,+}, \\ -1 & -k_{0,+}<k_{y}<-k_{\pi,+} \\ 0 & -k_{\pi,+}<k_{y}<k_{\pi,+} \\ 1 & k_{\pi,+}<k_{y}<k_{0,+} \\ 0 & k_{0,+}<k_{y}<k_{0,-} \\ 1 & k_{0,-}<k_{y}<k_{\pi,-}, \\ 2 & k_{\pi,-}<k_{y}<\pi .\end{cases}
$$

The winding number for $0<\mu<\frac{2 t}{\lambda} \sqrt{\lambda^{2}-4 t^{2}}$ [region H in Fig. 14] is

$$
W\left(k_{y}\right)= \begin{cases}-2 & -\pi<k_{y}<-k_{0,-}, \\ -1 & -k_{0,-}<k_{y}<-k_{\pi,-}, \\ 0 & -k_{\pi,-}<k_{y}<-k_{0,+}, \\ -1 & -k_{0,+}<k_{y}<-k_{\pi,+}, \\ 0 & -k_{\pi,+}<k_{y}<k_{\pi,+}, \\ 1 & k_{\pi,+}<k_{y}<k_{0,+}, \\ 0 & k_{0,+}<k_{y}<k_{\pi,-}, \\ 1 & k_{\pi,-}<k_{y}<k_{0,-}, \\ 2 & k_{0,-}<k_{y}<\pi .\end{cases}
$$

The winding number for $-2 t+\sqrt{4 t^{2}+\lambda^{2}}<\mu<+2 t+\sqrt{4 t^{2}+\lambda^{2}}$ [region I in Fig. 14] is

$$
W\left(k_{y}\right)= \begin{cases}-2 & -\pi<k_{y}<-k_{\pi,-} \\ -1 & -k_{\pi,-}<k_{y}<-k_{\pi,+} \\ 0 & -k_{\pi,+}<k_{y}<k_{\pi,+} \\ 1 & k_{\pi,+}<k_{y}<k_{\pi,-} \\ 2 & k_{\pi,-}<k_{y}<\pi .\end{cases}
$$

\section{Appendix C: Odd-frequency spin-triplet $s$-wave pair amplitude for semi-infinite system}

Frequency dependence of the odd-frequency spin-triplet $s$-wave pair amplitude at the surface of the semi-infinite $\mathrm{SC}$ is plotted for $S_{z}=1,0$ and -1 in Fig. [15.

In Figs. 15 (a) to (d), the odd-frequency spin-triplet $s$-wave pair amplitude for the spin-triplet $p_{x}$-wave superconductor are shown. Without the RSOC, $P_{S_{z}}^{\text {triplet }}\left(\omega_{n}\right)$ with $S_{z}= \pm 1$ is zero due to the spin-rotational symmetry but $P_{S_{z}}^{\text {triplet }}\left(\omega_{n}\right)$ with $S_{z}=0$ has large value due to translational symmetry braking and it diverges near $\omega_{n}=0$ [Fig. 15 (a)]. At $\lambda / \Delta_{0}=0.1$ shown in Fig. 15 (b), $P_{S_{z}}^{\text {triplet }}\left(\omega_{n}\right)$ with $S_{z}=0$ greatly suppressed and $P_{S_{z}}^{\text {triplet }}\left(\omega_{n}\right)$ with $S_{z}= \pm 1$ has non-zero value due to the spin-rotational symmetry breaking. $P_{S_{z}}^{\text {triplet }}\left(\omega_{n}\right)$ diverges very close to $k_{y}=0$ since there is ZESABS at $k_{y}=0$. For $\lambda / \Delta_{0} \gtrsim 1, P_{S_{z}}^{\text {triplet }}\left(\omega_{n}\right)$ with $S_{z}=0$ almost vanishes [Figs. 15] (c) and (d)] and all the components of $P_{S_{z}}^{\text {triplet }}\left(\omega_{n}\right)$ are almost zero for $\lambda / \Delta_{0} \gtrsim 10$ shown in Fig. 15(d). For $\lambda / \Delta_{0}=10$, we cannot see divergent behavior for $\left|\omega_{n} / \Delta_{0}\right|>5 \times 10^{-6}$.

In Figs. 15 (e) to (h), the odd-frequency spin-triplet $s$-wave pair amplitude for the spin-singlet $d_{x y}$-wave superconductor are shown. $P_{S_{z}}^{\text {triplet }}\left(\omega_{n}\right)$ is zero for $\lambda / \Delta_{0}=0$ due to the spin-rotational symmetry [Fig. 15] (e)]. For $\lambda / \Delta_{0}>0$,

absolute value of $P_{S_{z}}^{\text {triplet }}\left(\omega_{n}\right)$ increases as the increase of $\lambda / \Delta_{0}$ [Figs. 15 (f) to (h)]. The absolute value of $P_{S_{z}}^{\text {triplet }}\left(\omega_{n}\right)$ with $S_{z}= \pm 1$ is larger than that with $S_{z}=0$ since the even-frequency spin-triplet with $S_{z}=0$ pair amplitude is absent in the bulk [see also the Appendix 国. In these graphs, the absolute value of $P_{S_{z}}^{\text {triplet }}\left(\omega_{n}\right)$ increases as the increase of $\lambda$. 


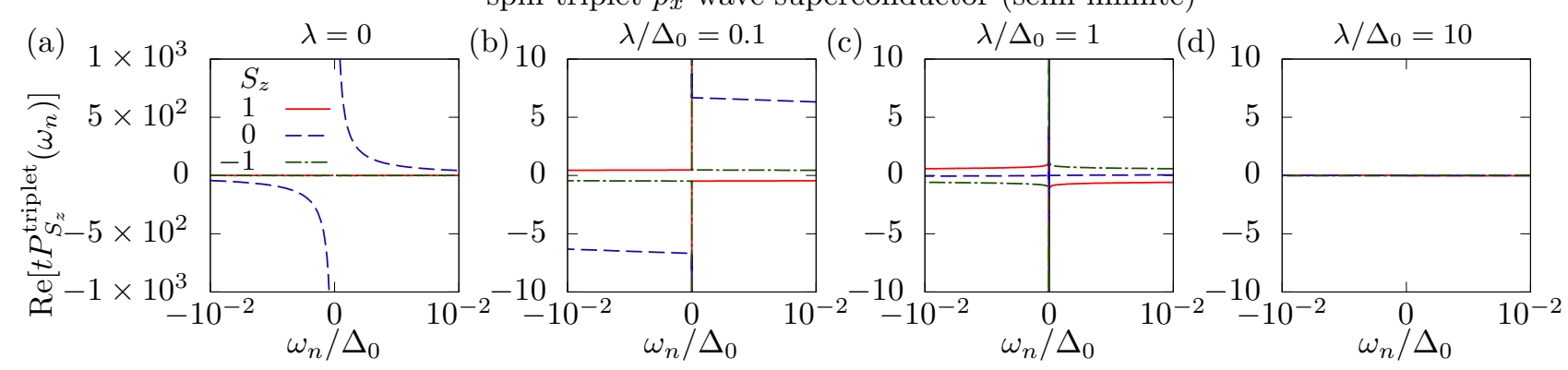

(e)

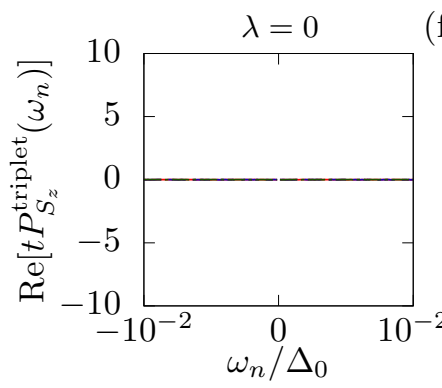

spin-singlet $d_{x y}$-wave superconductor (semi-infinite)
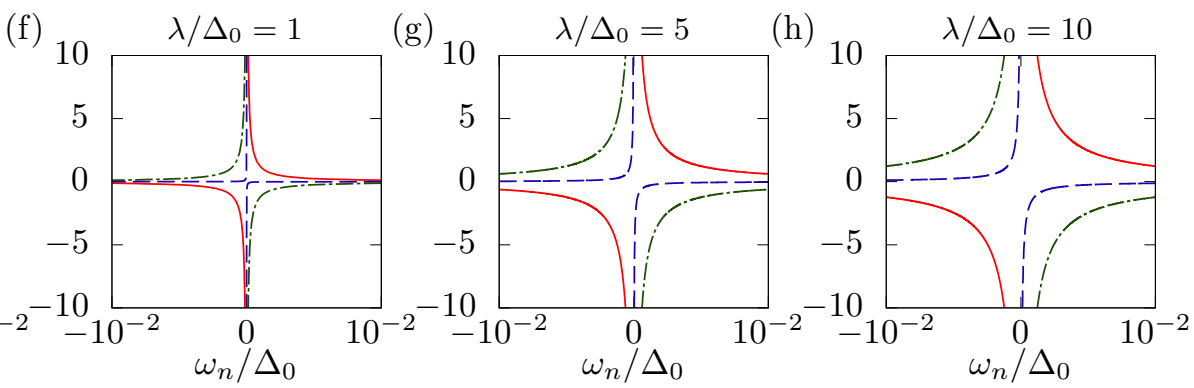

FIG. 15. The real part of the odd-frequency spin-triplet $s$-wave pair amplitude $P_{S_{z}}^{\text {triplet }}\left(\omega_{n}\right)$ is plotted as a function of $\omega_{n}$ for $S_{z}=-1,0,1$. The imaginary part of $P_{S_{z}}^{\text {triplet }}\left(\omega_{n}\right)$ is zero within numerical accuracy. The odd-frequency pair amplitude of the spin-triplet $p_{x}$-wave superconductor is shown in (a) $\lambda / \Delta_{0}=0$, (b) 0.1 , (c) 1 and (d) 10 . The odd-frequency pair amplitude of the spin-singlet $d_{x y}$-wave superconductor is shown in (e) $\lambda / \Delta_{0}=0$, (f) 1, (g) 5 and (h) 10 . In all cases, we set $\Delta_{0} / t=0.01$.

\section{Appendix D: Odd-frequency spin-singlet $p_{y}$-wave pair amplitude for spin-singlet $d_{x y}$-wave superconductor}

In Fig. 16, the odd-frequency spin-singlet $p_{y}$-wave pair amplitude at the surface is shown for the semi-infinite spinsinglet $d_{x y}$-wave superconductor. The definition of the odd-frequency spin-singlet $p_{y}$-wave pair amplitude is given by

$$
P_{p_{y}}^{\text {singlet }}\left(\omega_{n}\right)=\frac{1}{2 N_{k_{y}}} \sum_{k_{y}}\left[\hat{F}_{j_{x}=1, \uparrow, \downarrow}\left(i \omega_{n}, k_{y}\right)-\hat{F}_{j_{x}=1, \downarrow, \uparrow}\left(i \omega_{n}, k_{y}\right)\right] \sin k_{y}
$$

where $\hat{F}_{j_{x}, \sigma, \sigma^{\prime}}\left(i \omega_{n}, k_{y}\right)$ is the anomalous Green's function given by Eq. (17). The amplitude does not almost depend on the value of $\lambda$.

spin-singlet $d_{x y}$-wave superconductor (semi-infinite)

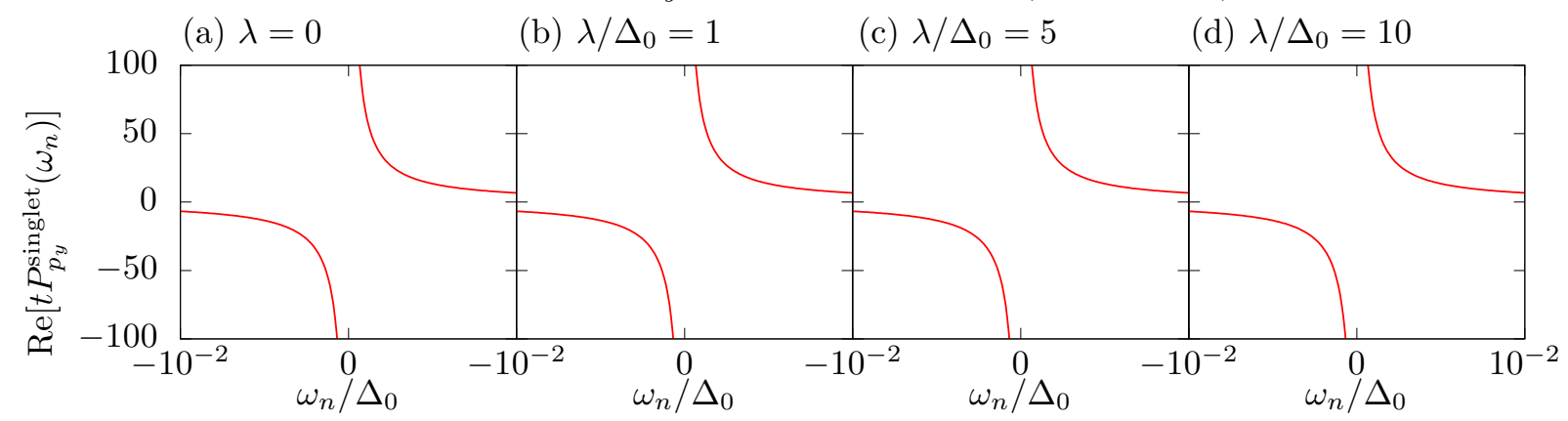

FIG. 16. The real part of the odd-frequency spin-singlet $p_{y}$-wave pair amplitude is plotted as a function of $\omega_{n} / \Delta_{0}$ for (a) $\lambda / \Delta_{0}=0$, (b) 1 , (c) 5 , and (d) 10 with $\Delta_{0} / t=0.01$. The imaginary part of the odd-frequency spin-singlet $p_{y}$-wave pair amplitude is zero within numerical accuracy. 


\section{Appendix E: Anomalous Green's function for $d_{x y}$-wave superconductor for bulk system}

The bulk Hamiltonian with $d_{x y}$-wave superconductor is given by

$$
\begin{aligned}
& H_{\mathrm{bulk}}=\sum_{\mathbf{k}} \bar{C}_{\mathbf{k}}^{\dagger} H(\mathbf{k}) \bar{C}_{\mathbf{k}} \\
& H(\mathbf{k})=\left(\begin{array}{cc}
E(\mathbf{k}) & \Delta(\mathbf{k}) \\
\Delta^{\dagger}(\mathbf{k}) & E^{\mathrm{T}}(-\mathbf{k})
\end{array}\right), \\
& E(\mathbf{k})=E_{0} \sigma_{0}+E_{1} \sigma_{1}+E_{2} \sigma_{2} \text {, } \\
& E_{0}=\left[-2 t\left(\cos k_{x}+\cos k_{y}\right)-\mu\right] \sigma_{0}, \\
& E_{1}=+\lambda \sin k_{y} \sigma_{1} \text {, } \\
& E_{2}=\lambda \sin k_{x} \sigma_{2} \text {, } \\
& \Delta(\mathbf{k})=\Delta_{0} \sin k_{x} \sin k_{y} i \sigma_{2} \\
& =\tilde{\Delta} \sigma_{2} \text {, } \\
& \bar{C}_{\mathrm{k}}=\left(c_{\uparrow, \mathbf{k}}, c_{\downarrow, \mathbf{k}}, c_{\uparrow,-\mathbf{k}}^{\dagger}, c_{\downarrow,-\mathbf{k}}^{\dagger}\right) .
\end{aligned}
$$

The anomalous Green's function for bulk system with the spin-singlet $d_{x y}$-wave superconductor is given by

$$
\begin{aligned}
G_{12}(z, \mathbf{k}) & =\frac{1}{\alpha_{0}^{2}-\alpha_{2}^{2}-\alpha_{3}^{2}}\left(\alpha_{0} \sigma_{0}-\alpha_{2} \sigma_{2}-\alpha_{3} \sigma_{3}\right), \\
\alpha_{0} & =\frac{2 E_{0} E_{2}}{\tilde{\Delta}} \\
\alpha_{2} & =-\tilde{\Delta}^{*}+\frac{z^{2}}{\tilde{\Delta}}-\frac{1}{\tilde{\Delta}}\left(E_{0}^{2}+E_{1}^{2}+E_{2}^{2}\right), \\
\alpha_{3} & =-\frac{2 i E_{0} E_{1}}{\tilde{\Delta}}
\end{aligned}
$$

with

$$
\begin{aligned}
& G(z, \mathbf{k})=[z-H(\mathbf{k})]^{-1} \\
& G(z, \mathbf{k})=\left(\begin{array}{ll}
G_{11}(z, \mathbf{k}) & G_{12}(z, \mathbf{k}) \\
G_{21}(z, \mathbf{k}) & G_{22}(z, \mathbf{k})
\end{array}\right)
\end{aligned}
$$

where $G_{i j}(z, \mathbf{k})(i, j=1,2)$ is a $2 \times 2$ matrix. From Eq. (E10), we can see that the spin-triplet component with $S_{z}=0$ is absent since $S_{z}=0$ component is proportional to $\sigma_{1}$.

\section{Appendix F: Matrix elements of Green's function for SC/DN junction}

In this Appendix, we explain the derivation of the Green's function in the DN in DN/superconductor junctions where we use super unit cell in the $y$-direction $\left(-\pi / L_{y}<k_{y} \leq \pi / L_{y}\right)$ with periodic boundary condition. The Hamiltonian in the DN is

$$
\mathcal{H}_{\mathrm{DN}}^{l_{s}}=\sum_{k_{y}} \tilde{C}^{\dagger}\left(k_{y}\right)\left(\begin{array}{cccccc}
\tilde{u}^{(-49), l_{s}} & \tilde{t} & & & \\
& \tilde{t}^{\dagger} & \tilde{u}^{(-48), l_{s}} & \tilde{t} & & \\
& \tilde{t}^{\dagger} & \tilde{u}^{(-47), l_{s}} & \tilde{t} & \\
& & & \ddots & \\
& & & & & \\
& & & & \tilde{t}^{\dagger} & \tilde{u}^{(0), l_{s}}
\end{array}\right) \tilde{C}\left(k_{y}\right),
$$


with $4 L_{y} \times 4 L_{y}$ matrices

$$
\begin{aligned}
& \tilde{u}^{\left(j_{x}\right), l_{s}}=\left(\begin{array}{cccccc}
A_{1}^{\left(j_{x}, 1\right), l_{s}} & A_{2} & \hat{0} & \ldots & \hat{0} & A_{2}^{\dagger} \\
A_{2}^{\dagger} & A_{1}^{\left(j_{x}, 2\right), l_{s}} & A_{2} & \hat{0} & & \hat{0} \\
\hat{0} & A_{2}^{\dagger} & A_{1}^{\left(j_{x}, 3\right), l_{s}} & A_{2} & \hat{0} & \vdots \\
\vdots & & & \ddots & & \hat{0} \\
\hat{0} & & \hat{0} & A_{2}^{\dagger} & A_{1}^{\left(j_{x}, L_{y}-1\right), l_{s}} & A_{2} \\
A_{2} & \hat{0} & \ldots & \hat{0} & A_{2}^{\dagger} & A_{1}^{\left(j_{x}, L_{y}\right), l_{s}}
\end{array}\right) \text {, } \\
& \tilde{t}=\left(\begin{array}{cccccc}
B_{1} & B_{12} & \hat{0} & \cdots & \hat{0} & B_{21} \\
B_{21} & B_{1} & B_{12} & \hat{0} & & \hat{0} \\
\hat{0} & B_{21} & B_{1} & B_{12} & \hat{0} & \vdots \\
\vdots & & & \ddots & & \hat{0} \\
\hat{0} & \ldots & \hat{0} & B_{21} & B_{1} & B_{12} \\
B_{12} & \hat{0} & \ldots & \hat{0} & B_{21} & B_{1}
\end{array}\right),
\end{aligned}
$$

and

$$
\begin{aligned}
\tilde{C}\left(k_{y}\right) & =\left(\tilde{\boldsymbol{c}}_{(-49,1), k_{y}}, \tilde{\boldsymbol{c}}_{(-49,2), k_{y}}, \cdots, \tilde{\boldsymbol{c}}_{\left(-49, L_{y}\right), k_{y}}, \tilde{\boldsymbol{c}}_{(-48,1), k_{y},}, \tilde{\boldsymbol{c}}_{(-48,2), k_{y}}, \cdots, \tilde{\boldsymbol{c}}_{\left(0, L_{y}\right), k_{y}},\right)^{\mathrm{T}}, \\
\tilde{\boldsymbol{c}}_{\mathbf{j}, k_{y}} & =\left(c_{\mathbf{j}, k_{y}, \uparrow}, c_{\mathbf{j}, k_{y}, \downarrow}, c_{\mathbf{j},-k_{y}, \uparrow}^{\dagger}, c_{\mathbf{j},-k_{y}, \downarrow}^{\dagger}\right) .
\end{aligned}
$$

Here, $A_{1}^{\left(j_{x}, y_{y}\right), l_{s}}$ and $A_{2}$ are $4 \times 4$ matrices given by

$$
\begin{aligned}
& A_{1}^{\left(j_{x}, j_{y}\right), l_{s}}=\left(\begin{array}{llll}
-\mu+V_{\left(j_{x}, j_{y}\right)}^{l_{s}} & & & \\
& -\mu+V_{\left(j_{x}, j_{y}\right)}^{l_{s}} & & \\
& \mu-V_{\left(j_{x}, j_{y}\right)}^{l_{s}} & \\
& & \mu-V_{\left(j_{x}, j_{y}\right)}^{l_{s}}
\end{array}\right), \\
& A_{2}=\left(\begin{array}{cccc}
-t e^{i k_{y}} & -i \lambda e^{i k_{y} / 2} & & \\
-i \lambda e^{i k_{y} / 2} & -t e^{i k_{y}} & & \\
& & t e^{i k_{y}} & -i \lambda e^{i k_{y}} / 2 \\
& & -i \lambda e^{i k_{y}} / 2 & t e^{i k_{y}}
\end{array}\right),
\end{aligned}
$$

where $-t<V_{\mathbf{j}}^{l_{s}}<t$ is a random potential at $\mathbf{j}=\left(j_{x}, j_{y}\right)$-th site. $B_{1}, B_{12}$ and $B_{21}$ are $4 \times 4$ matrices and they are given by

$$
\begin{aligned}
B_{1} & =\left(\begin{array}{cccc}
-t & \lambda / 2 & & -i \Delta_{0} / 2 \\
-\lambda / 2 & -t & -i \Delta_{0} / 2 & \\
& -i \Delta_{0} / 2 & t & -\lambda / 2 \\
-i \Delta_{0} / 2 & & \lambda / 2 & t
\end{array}\right), \\
B_{12} & =B_{21}=\hat{0},
\end{aligned}
$$

for the spin-triplet $p_{x}$-wave superconductor. For the spin-singlet $d_{x y}$-wave superconductor, $B_{1}, B_{12}$ and $B_{21}$ are given by

$$
\begin{aligned}
B_{1} & =\left(\begin{array}{cccc}
-t & \lambda / 2 & & \\
-\lambda / 2 & -t & & \\
& & t & -\lambda / 2 \\
\lambda / 2 & t
\end{array}\right), \\
B_{12} & =-B_{21}^{*}=\left(\begin{array}{c}
\Delta_{0} e^{i k_{y} / 4} \\
-\Delta_{0} e^{i k_{y}} / 4 \\
\Delta_{0} e^{i k_{y}} / 4
\end{array}\right) .
\end{aligned}
$$


The Green's function in the DN is then obtained as

$$
\tilde{g}_{j_{x}}^{\mathrm{DN}, l_{s}}\left(z, j_{y}, j_{y}^{\prime}, k_{y}\right)=\left\{\left[\tilde{g}_{j_{x}}^{(\mathrm{L}), l_{s}}\left(z, j_{y}, j_{y}^{\prime}, k_{y}\right)\right]^{-1}-\tilde{t}_{j_{x}+1}^{(\mathrm{R}), l_{s}}\left(z, j_{y}, j_{y}^{\prime}, k_{y}\right) \tilde{t}^{\dagger}\right\}^{-1},
$$

where $\tilde{g}_{j_{x}}^{(\mathrm{L}), l_{s}}\left(z, j_{y}, j_{y}^{\prime}, k_{y}\right)$ and $\tilde{g}_{j_{x}+1}^{(\mathrm{R}), l_{s}}\left(z, j_{y}, j_{y}^{\prime}, k_{y}\right)$ are given by

$$
\begin{aligned}
& \tilde{g}_{j_{x}, l_{s}}^{(\mathrm{L})}\left(z, j_{y}, j_{y}^{\prime}, k_{y}\right)=\left(X_{l_{x}=j_{x}}^{l_{s}} \ldots X_{l_{x}=-48}^{l_{s}} X_{l_{x}=-49}^{l_{s}}\right) \bullet \\
& \tilde{g}_{j_{x}+1}^{(\mathrm{R}), l_{s}}\left(z, j_{y}, j_{y}^{\prime}, k_{y}\right)=\left(Y_{l_{x}=j_{x}+1}^{l_{s}} Y_{l_{x}=j_{x}+2}^{l_{s}} \ldots Y_{l_{x}=0}^{l_{s}}\right) \tilde{G}^{\infty}\left(z, j_{y}, j_{y}^{\prime}, k_{y}\right),
\end{aligned}
$$

with $4 L_{y} \times 4 L_{y}$ matrix $\tilde{0}$ where all the elements of $\tilde{0}$ are zero. Here $X_{l_{x}}^{l_{s}}$ and $Y_{l_{x}}^{l_{s}}$ are given by

$$
\begin{aligned}
X_{l_{x}}^{l_{s}} & =\left(\begin{array}{cc}
\tilde{0} & \tilde{t}^{-1} \\
-\tilde{t}^{\dagger} & {\left[z-\tilde{u}^{\left(l_{x}\right), l_{s}}\right] \tilde{t}^{-1}}
\end{array}\right), \\
Y_{l_{x}}^{l_{s}} & =\left(\begin{array}{cc}
\tilde{0} & \left(\tilde{t}^{\dagger}\right)^{-1} \\
-\tilde{t} & {\left[z-\tilde{u}^{\left(l_{x}\right), l_{s}}\right]\left(\tilde{t}^{\dagger}\right)^{-1}}
\end{array}\right),
\end{aligned}
$$

and $\tilde{G}^{\infty}\left(z, j_{y}, j_{y}^{\prime}, k_{y}\right)$ is given by

$$
\tilde{G}^{\infty}\left(z, j_{y}, j_{y}^{\prime}, k_{y}\right)=\sum_{m=0,1, \ldots, N_{y}-1} e^{i \frac{2 \pi m}{N_{y}}\left(j_{y}-j_{y}^{\prime}\right)} G_{j_{x}=1}^{\infty}\left(z, k_{y}+\frac{2 \pi m}{N_{y}}\right)
$$

where $k_{y}$ is $k_{y}=-\frac{\pi\left(N_{y}-1\right)}{N_{y} L_{y}},-\frac{\pi\left(N_{y}-2\right)}{N_{y} L_{y}}, \ldots, \frac{\pi}{L_{y}} . G_{j_{x}=1}^{\infty}\left(z, k_{y}^{\prime}\right)$ with $k_{y}^{\prime}=k_{y}+2 \pi m / N_{y}$ is given by Eq. (A20).

\section{Appendix G: Size dependence of LDOS in DN for spin-singlet $d_{x y}$-wave superconductor junction}

In this Appendix, we check the size and the number of sample dependence of LDOS at the center of the DN $\left(j_{x}=-L_{x} / 2\right.$ where $L_{x}$ is a length of the DN in the $x$-direction) for the spin-singlet $d_{x y}$-wave superconductor junction with $\lambda / \Delta_{0}=50$.

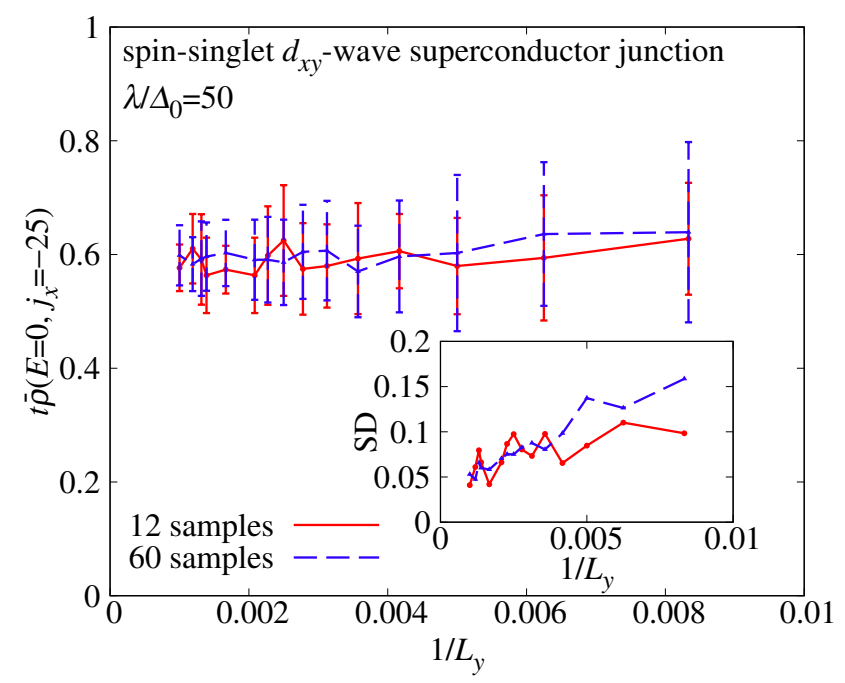

FIG. 17. Zero energy LDOS at the center in the DN $\bar{\rho}\left(E=0, j_{x}=-25\right)$ is plotted as a function of the inverse of the size in the $y$-direction $1 / L_{y}$. Standard deviation (SD) estimated by 12 and 60 samples is also plotted. The length of the DN in the $x$-direction is $L_{x}=50$. SD (error bar in main panel) is plotted in the inset as a function of $1 / L_{y}$.

In Fig. 17, we plot LDOS at zero-energy averaged over 12 and 60 samples as a function of $1 / L_{y}$. As we can see in this figure, the extrapolated value of the ZEP for $L_{y} \rightarrow \infty$ is much larger than the background value $(\sim 0.2)$. In the 


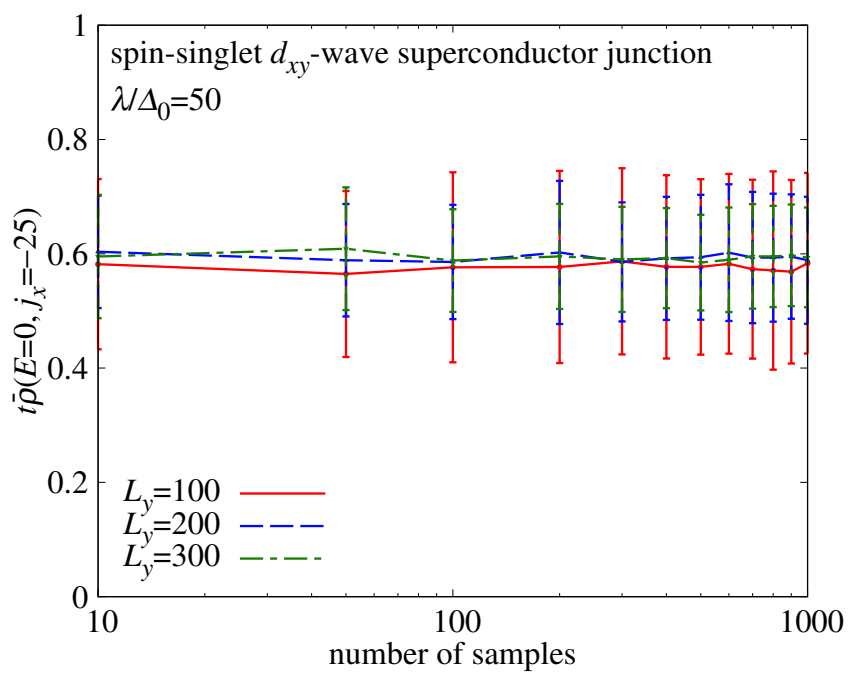

FIG. 18. Zero energy LDOS at the center in the DN $\bar{\rho}\left(E=0, j_{x}=-25\right)$ is plotted as a function of the number of samples for $L_{y}=100,200$ and 300. Standard deviation is also plotted. The length of the DN is $L_{x}=50$.

inset of Fig. 17 we plot the corresponding standard deviation. Although the magnitude of the standard deviation is not small, it decreases with the increase of $L_{y}$.

In Fig. 18, we plot the value of ZEP of LDOS as a function of the number of samples. The height of ZEP is almost independent of the number of samples for all values of $L_{y}$. The value of standard deviation becomes smaller with the increase of $L_{y}$.

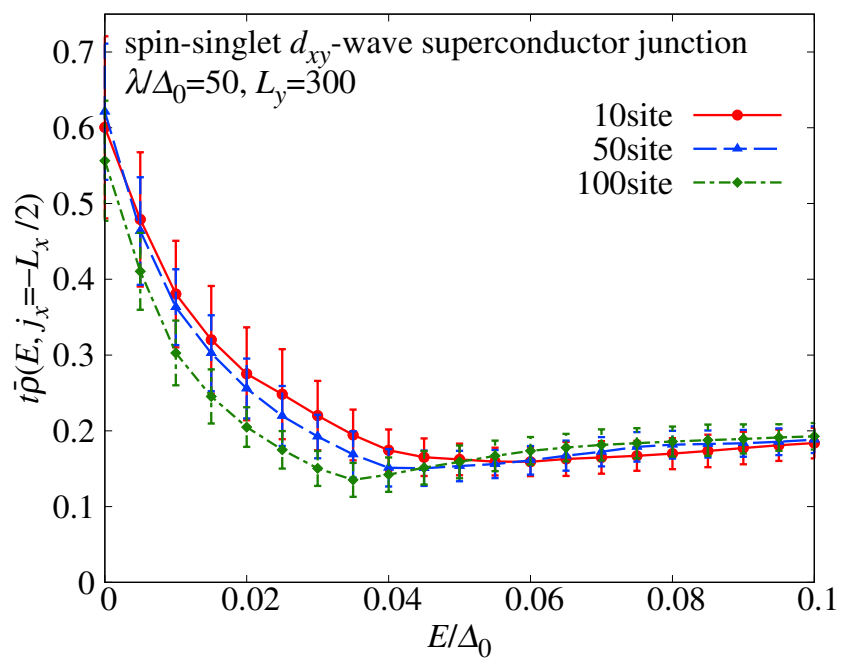

FIG. 19. LDOS at the center in the $\mathrm{DN} \bar{\rho}\left(E, j_{x}=-L_{x} / 2\right)$ for several length of the DN $\left(L_{x}=10,50\right.$ and 100$)$ is plotted as a function of energy with 60 samples and $L_{y}=300$.

We also check $L_{x}$ dependence of LDOS at the center of the DN where $L_{x}$ is a length of the DN in the $x$-direction. In Fig. 19] we change $L_{x}$ from 10 to 100 and plot LDOS at $x=-L_{x} / 2$. Although, it is difficult to estimate the peak width of LDOS due to the large value of standard deviation, it decreases with the increase of $L_{x}$.

\section{Appendix H: Spatial dependence of odd-frequency pair amplitude for the DN/SC junction}

In this Appendix, we discuss the spatial dependence of the odd-frequency spin-triplet $s$-wave pair amplitude for the $\mathrm{DN} / d_{x y}$-wave superconductor junction. The odd-frequency spin-triplet $s$-wave pair amplitudes at each site $\left(j_{x}\right)$ given 
by

$$
\begin{aligned}
P_{j_{x}, S_{z}=1}^{\text {triplet }}\left(\omega_{n}\right) & =P_{j_{x}, \uparrow \uparrow}\left(\omega_{n}\right), \\
P_{j_{x}, S_{z}=1}^{\text {triplet }}\left(\omega_{n}\right) & =\frac{1}{\sqrt{2}}\left[P_{j_{x}, \uparrow, \downarrow}\left(\omega_{n}\right)+P_{j_{x}, \downarrow, \uparrow}\left(\omega_{n}\right)\right], \\
P_{j_{x}, S_{z}=-1}^{\text {triplet }}\left(\omega_{n}\right) & =P_{j_{x}, \downarrow, \downarrow}\left(\omega_{n}\right),
\end{aligned}
$$

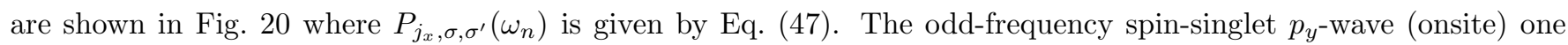
given by

$$
\begin{aligned}
P_{j_{x}, p_{y}}^{\text {singlet }}\left(\omega_{n}\right)=\frac{1}{2 N_{k_{y}} L_{y} N_{\text {sample }}} \sum_{k_{y}, j_{y}, l_{s}} & \frac{1}{2 \sqrt{2}}\left\{\left[\hat{f}_{j_{x}, \uparrow, \downarrow}^{l_{s}}\left(i \omega_{n}, j_{y}, j_{y}+1, k_{y}\right)-\hat{f}_{j_{x}, \downarrow \uparrow \uparrow}^{l_{s}}\left(i \omega_{n}, j_{y}, j_{y}+1, k_{y}\right)\right]\right. \\
- & {\left.\left[\hat{f}_{j_{x}, \uparrow, \downarrow}^{l_{s}}\left(-i \omega_{n}, j_{y}, j_{y}+1, k_{y}\right)-\hat{f}_{j_{x}, \downarrow, \uparrow}^{l_{s}}\left(-i \omega_{n}, j_{y}, j_{y}+1, k_{y}\right)\right]\right\} e^{-i k_{y}}, }
\end{aligned}
$$

is shown in Fig. 21] Here $\hat{f}_{j_{x}, \sigma, \sigma^{\prime}}^{l_{s}}\left(z, j_{y}, j_{y}^{\prime}, k_{y}\right)$ is the anomalous Green's function of $\tilde{g}_{j_{x}}^{\mathrm{DN}, l s}\left(z, j_{y}, j_{y}^{\prime}, k_{y}\right)$ given by Eq. (F12).

\section{spin-singlet $d_{x y}$-wave superconductor $\mathrm{DN} / \mathrm{SC}$ junction}
(a) $S_{z}=1$
(b) $S_{z}=0$
(c) $S_{z}=-1$

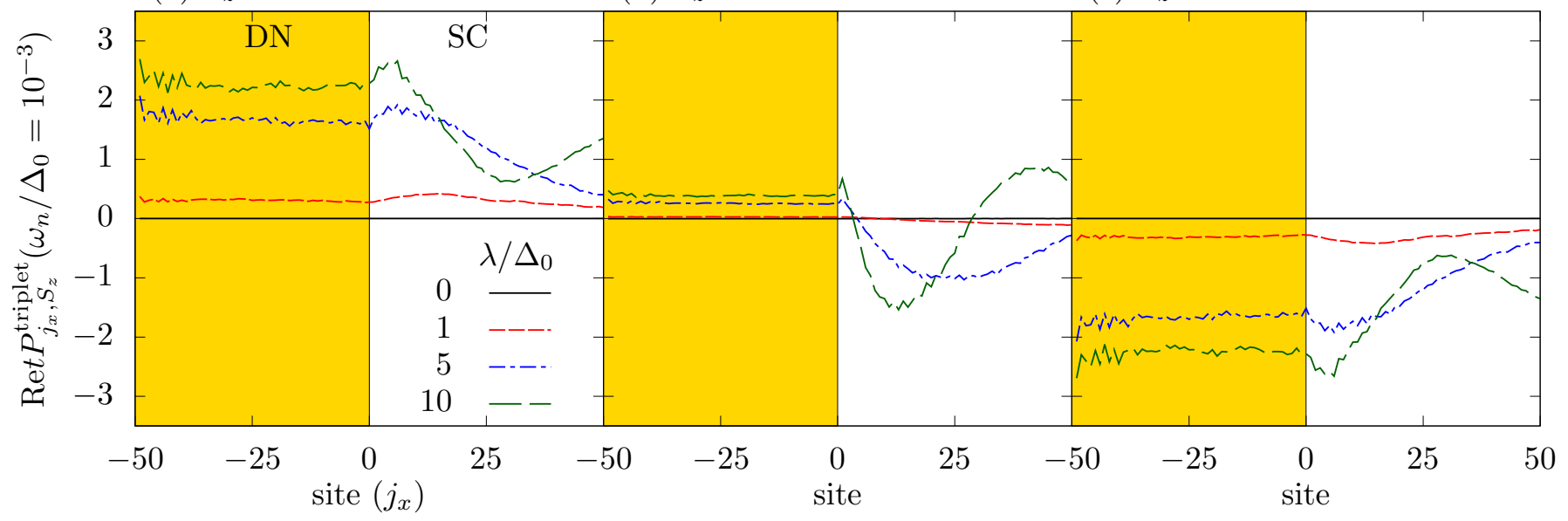

FIG. 20. The real part of the odd-frequency spin-triplet $s$-wave pair amplitude with $\omega_{n} / \Delta_{0}=10^{-3}\left(\Delta_{0} / t=0.01\right)$ is plotted as a function of site $\left(j_{x}\right)$ for (a) $S_{z}=1$, (b) $S_{z}=0$ and (c) $S_{z}=-1$ with several $\lambda\left(\lambda / \Delta_{0}=0,1,5\right.$, and 10) for the DN/spin-singlet $d_{x y}$-wave superconductor junction. The imaginary part of the odd-frequency spin-triplet $s$-wave pair amplitude is zero within numerical accuracy.

The odd-frequency spin-triplet $s$-wave pair amplitude $P_{j_{x}, S_{z}}^{\text {triplet }}\left(\omega_{n} / \Delta_{0}=10^{-3}\right)$ is almost constant in the DN and $P_{j_{x}, S_{z}=1}\left(\omega_{n}\right)+P_{j_{x}, S_{z}=-1}\left(\omega_{n}\right)=0$ holds within numerical accuracy. The odd-frequency spin-singlet $p_{y}$-wave pair amplitude rapidly decays in the DN since $p$-wave pair amplitudes are fragile against impurity scattering. $p_{x}$-wave components are almost zero in the DN and the SC.

\section{Appendix I: Surface roughness for spin-singlet $d_{x y}$-wave superconductor}

In order to study the effect of roughness, we study a thin diffusive layer attached to the surface of $d_{x y}$-wave superconductor shown in Fig. 22. The effect of diffusive scattering on the SABS has been studied within quasiclassical approximation [91, 92]. Without RSOC, induced odd-frequency pair amplitude is suppressed since it has an odd parity [93]. On the other hand, in the presence of the RSOC, the odd-frequency spin-triplet $s$-wave pair amplitude is induced near the surface [35]. Here, we take into account of the spatial depletion of the pair potential near the surface. The assumed spatial dependence is shown in Eq. (133). The Hamiltonian is given by 
spin-singlet $d_{x y}$-wave superconductor DN/SC junction

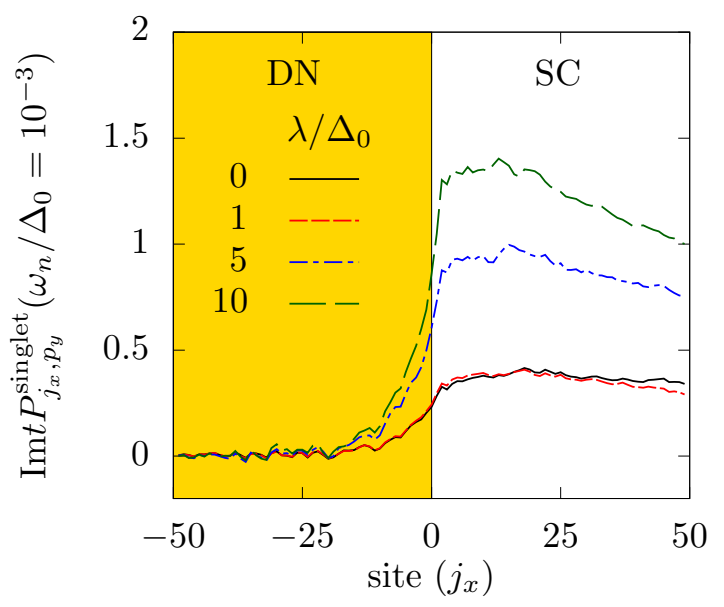

FIG. 21. The imaginary part of the odd-frequency spin-singlet $p_{y}$-wave pair amplitude with $\omega_{n} / \Delta_{0}=10^{-3}\left(\Delta_{0} / t=0.01\right)$ is plotted as a function of site $\left(j_{x}\right)$ with several $\lambda\left(\lambda / \Delta_{0}=0,1,5\right.$, and 10) for the DN/spin-singlet $d_{x y}$-wave superconductor junction. The real part of the odd-frequency spin-singlet $p_{y}$-wave pair amplitude is zero within numerical accuracy.

spin-singlet $d_{x y}$-wave superconductor

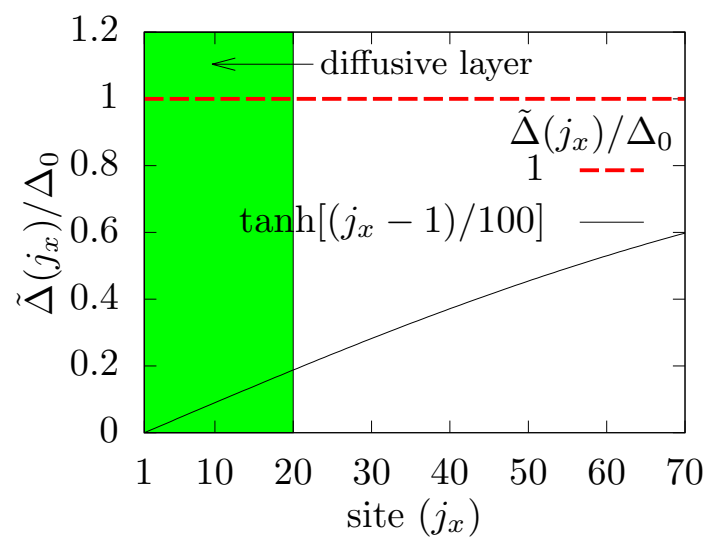

FIG. 22. Schematic of the SC with impurity potential $\left(1 \leq j_{x} \leq N_{\mathrm{d}}=20\right)$. Green colored region indicates the area with impurity potential. Pair potential $\tilde{\Delta}\left(j_{x}\right)$ is also plotted as a function of site $\left(j_{x}\right)$.

$$
\begin{aligned}
\mathcal{H} & =\mathcal{H}_{t}+\sum_{1 \leq j_{x} \leq N_{\mathrm{d}}, j_{y}} V_{\mathbf{j}} n_{\mathbf{j}}+\mathcal{H}_{\mathrm{SO}}-\mu \sum_{\mathbf{j}} n_{\mathbf{j}}+\tilde{\mathcal{H}}_{\Delta}, \\
\tilde{\mathcal{H}}_{\Delta} & =-\frac{1}{2} \sum_{\mathbf{j}} \tilde{\Delta}\left(j_{x}\right)\left(c_{\mathbf{j}, \uparrow}^{\dagger} c_{\mathbf{j}+\mathbf{e}_{x}+\mathbf{e}_{y}, \downarrow}^{\dagger}-c_{\mathbf{j}, \downarrow}^{\dagger} c_{\mathbf{j}+\mathbf{e}_{x}+\mathbf{e}_{y}, \uparrow}^{\dagger}-c_{\mathbf{j}, \uparrow}^{\dagger} c_{\mathbf{j}+\mathbf{e}_{x}-\mathbf{e}_{y}, \downarrow}^{\dagger}+c_{\mathbf{j}, \downarrow}^{\dagger} c_{\mathbf{j}+\mathbf{e}_{x}-\mathbf{e}_{y}, \uparrow}^{\dagger}\right), \\
\tilde{\Delta}\left(j_{x}\right) & =\left\{\begin{array}{l}
\Delta_{0}, \\
\Delta_{0} \tanh \left[\left(j_{x}-1\right) / 100\right],
\end{array}\right.
\end{aligned}
$$

In Eq. (I3), we take into account the fact that the coherence length is about 100 site $\left(\Delta_{0} / t=0.01\right)$. Here we impose impurity potential $V_{\mathbf{j}}$ for $1 \leq j_{x} \leq N_{\mathrm{d}}=20$. In Fig. 23, absolute value of the odd-frequency spin-triplet $s$-wave pair amplitude given by

$$
\left|P_{j_{x}}^{\text {triplet }}\left(\omega_{n}\right)\right|=\sum_{S_{z}=1,0,-1}\left|P_{j_{x}, S_{z}}^{\text {triplet }}\left(\omega_{n}\right)\right|
$$

is shown for $\lambda / \Delta_{0}=1,5$ and 10. Here $P_{j_{x}, S_{z}}^{\text {triplet }}\left(\omega_{n}\right)$ is given by Eqs. (H1) to (H3). From Fig. 23) we can conclude that the spatial dependence of the pair potential near the surface almost does not affect the magnitude of the odd-frequency 
spin-singlet $d_{x y}$-wave superconductor with diffusive layer

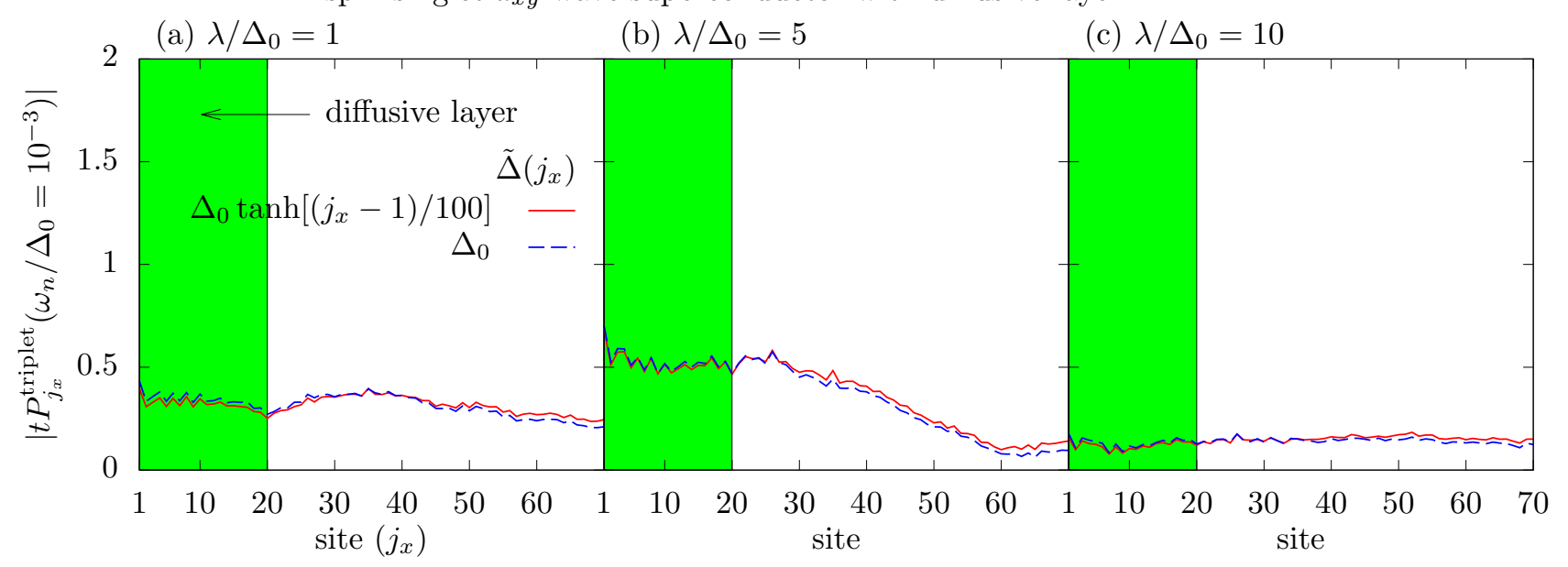

FIG. 23. The absolute value of the odd-frequency spin-triplet $s$-wave pair amplitude is plotted as a function of site $\left(j_{x}\right)$ for the spin-singlet $d_{x y}$-wave superconductor with diffusive layer (green colored region). $P^{\text {triplet }}\left(\omega_{n} / \Delta_{0}=10^{-3}\right)$ for $\tilde{\Delta}\left(j_{x}\right)=\Delta_{0}$ and $\Delta_{0} \tanh \left[\left(j_{x}-1\right) / 100\right]$ are shown for (a) $\lambda / \Delta_{0}=1$, (b) $\lambda / \Delta_{0}=5$ and (c) $\lambda / \Delta_{0}=10$.

spin-triplet $s$-wave pair amplitude as compared to the case with constant pair potential. 